\title{
Circuit
}

Musiques contemporaines

\section{De la résurgence du merveilleux}

L'exemple de L'Eau qui danse, la Pomme qui chante et l'Oiseau qui dit la vérité, un opéra de Gilles Tremblay et Pierre Morency

On the Resurgence of the "merveilleux"

The Example of L'Eau qui danse, la Pomme qui chante et l'Oiseau qui dit la vérité, an opera by Gilles Tremblay and Pierre Morency

\section{Cécile Auzolle}

Volume 20, numéro 3, 2010

Gilles Tremblay, ou le plain-chant contemporain

URI : https://id.erudit.org/iderudit/044859ar

DOI : https://doi.org/10.7202/044859ar

Aller au sommaire du numéro

Éditeur(s)

Les Presses de l'Université de Montréal

ISSN

1183-1693 (imprimé)

1488-9692 (numérique)

Découvrir la revue

Citer cet article

Auzolle, C. (2010). De la résurgence du merveilleux : l'exemple de L'Eau qui

danse, la Pomme qui chante et l'Oiseau qui dit la vérité, un opéra de Gilles

Tremblay et Pierre Morency. Circuit, 20(3), 9-42.

https://doi.org/10.7202/044859ar
Résumé de l'article

Après avoir situé l'opéra-féerie de Gilles Tremblay et Pierre Morency créé à Montréal en novembre 2009 dans le contexte historique de l'opéra merveilleux, il est mené une étude du livret dans son rapport au conte originel de Madame d'Aulnoy (1696) et à ses modèles, puis des spécificités musicales et scéniques de L'Eau qui danse, la Pomme qui chante et l'Oiseau qui dit la vérité. Il s'agit enfin de comprendre comment et pourquoi le merveilleux est utilisé par les artistes pour ouvrir la voie à un renouveau de l'art lyrique utilisant pleinement les acquis musicaux, théâtraux et spirituels de leur temps. 


\section{De la résurgence du merveilleux}

\section{L'exemple de L'Eau qui danse, la Pomme qui chante et l'Oiseau qui dit la vérité, un opéra de Gilles Tremblay et Pierre Morency}

Cécile Auzolle

"Qu'il soit religieux ou non n'a pas d'importance, l'émerveillement me paraît être à la source de tout, à la base de toute vie entre les êtres.»

Tremblay, 1994b, p. 11

L'opéra se nourrit de merveilleux depuis ses origines florentines, en particulier à travers les métamorphoses, transformations et miracles qui émaillent les livrets mythologiques d'Orphée, de Daphné ou d'Ariane. En France, le baroque musical, concordant avec le classicisme littéraire, voit à la fin du $\mathrm{XVII}^{\mathrm{e}}$ siècle le développement de la poétique du merveilleux qui «prolonge la vraisemblance historique et naturelle tout en la singeant» (Kinztler, 1997, p. 407), conférant à l'opéra un statut d' «hyper-théâtre» (ibid., p. 408) sur la scène duquel les questions essentielles se trouvent épurées. Si le théâtre sérieux éclot et se développe au XVIII ${ }^{\mathrm{e}}$, la fin de ce siècle et le XIX retrouvent le goût de la féerie (Buch, 1992; Martin, 2003) tandis que se déploie parallèlement une autre forme d'art officiel, comme en atteste, par exemple, la composition conjointe de La Flûte enchantée et de La Clémence de Titus par Mozart en 1791. Dans la seconde partie du XIX ${ }^{\mathrm{e}}$ siècle et au XX $\mathrm{X}^{\mathrm{e}}$ siècle, le perfectionnement des techniques théâtrales, notamment à partir de la découverte de l'électricité, de l'utilisation du cinéma puis des nouvelles technologies, favorise et encourage la réalisation des effets spectaculaires (Christout, 1982; Yon, 2006) et, partant, la composition d'œuvres féeriques. Enfin, en réaction au matérialisme des trente glorieuses, les artistes, et les musiciens en particulier suivant l'exemple rayonnant d'Olivier Messiaen (Boivin, 1995), 
1. Mes remerciements les plus chaleureux vont à Pauline Vaillancourt pour m'avoir confié les documents nécessaires à cette étude: le programme, le livret, la captation audiovisuelle, la réduction chant/piano et la partition d'orchestre de L'Eau qui danse... Pour les détails du spectacle et de la distribution: www.chantslibres. org/fr/productions/eau/

2. Der blaue Vogel (1998), créé au théâtre de Hagen (Allemagne): www.omm.de/veranstaltungen/ musiktheater19992000/HA-der-blauevogel.html/

3. Abrégé dans le texte en "Conte du Tsar Saltan...".

4. Créé à Moscou au théâtre Solodovnikov le 3 novembre 1900. réexplorent les mystères de la nature et d'une spiritualité animiste héritée de Bachelard et des philosophies orientales.

Élève de la classe de «Philosophie musicale» de Messiaen entre 1954 et 1957 (Boivin, 1995, p. 418-419), considéré par son maître comme «le plus grand compositeur canadien à l'heure actuelle [1971 et 1985] » (Samuel, 1999, p. 156 et p. 315) et fort d'une œuvre qui fait la part belle à la musique instrumentale, Gilles Tremblay (né en 1932) se tourne vers la forme la plus académique de théâtre musical: l'opéra. Il y insuffle son attrait pour la synthèse des sons et des mouvements de l'univers inscrite dans une profonde tradition française d'Aquin à l'école spectrale (Boivin, 2001, p. 484-511), héritage de ses années parisiennes. Unique réalisation lyrique du compositeur, L'Eau qui danse, la Pomme qui chante et l'Oiseau qui dit la vérité a été créée au Monument-National à Montréal le 19 novembre 2009 par la compagnie Chants libres.1. Gilles Tremblay s'appuie sur un livret du poète Pierre Morency qui allie l'évocation des éléments aristotéliciens à la structure dramaturgique du conte de Madame d'Aulnoy dans une langue à la fois poétique, aphoristique, symbolique et profondément musicale. L'Eau qui danse... est intrinsèquement représentative du style et des quêtes spirituelles et esthétiques de son compositeur: si son ancrage dans le merveilleux la rapproche des esthétiques baroque et romantique, son traitement musical n'est en rien redevable à une quelconque nostalgie puisée dans l'histoire de la féerie ou du Märchenoper.

L'Eau qui danse... s'inscrit dans la tradition des opéras à quête, en particulier La Flûte enchantée, Le Tsar Saltan, La Femme sans ombre ou encore Turandot, et rejoint la lignée des opéras adaptés de contes ou de féeries comme Cendrillon, Hänsel et Gretel, Le Rossignol, L'Enfant et les Sortilèges, La Petite Renarde rusée, Brundibar, Le Roi-Cerf, Le Songe d'une nuit d'été ou encore le récent Oiseau bleu de Harald Banter également composé d'après un conte de Madame d'Aulnoy². La parenté avec les opéras de Mozart et Strauss est indéniable dans le sens où les groupes de protagonistes sont à la recherche d'une identité et d'une humanité qu'il leur faudra conquérir au terme d'un parcours initiatique. Mais l'opéra le plus proche de L'Eau qui danse... demeure le Conte du Tsar Saltan, de son fils le célèbre et puissant héros Prince Guidon Saltanovitch, et de la belle Princesse-Cygne $e^{3}$ de RimskyKorsakov4: les deux œuvres puisent aux mêmes sources, décrivant le périple de personnages injustement écartés de leur destin par la jalousie qui doivent reconquérir leur légitimité en traversant des épreuves. Ces sources sont réécrites et interprétées en vertu de cultures et d'époques données, dans un foisonnement imaginaire tropique pour le Russe et affûté pour le Québécois. 
Comment, au début du XXI ${ }^{\mathrm{e}}$ siècle, transformer en livret un conte venu des Mille et une nuits et maintes fois réécrit? Quels choix musicaux effectuer pour accompagner le cheminement des protagonistes et la révélation des mécanismes de bien, de mal, d'amour et d'honneur qui régissent l'organisation sociale dans la perspective d'une mise en scène? Enfin quelle place est donnée au merveilleux dans cette œuvre et comment s'insère-t-elle dans la création lyrique actuelle? Telles sont les questions auxquelles cette étude tentera de répondre, examinant tout à tour les sources du livret, son traitement musical et enfin l'expression du merveilleux dans la partition comme dans la mise en scène de la création.

\section{Du conte à la féerie}

\section{De l'orient des Mille et une nuits au Canada du Xxı siècle, en passant par la France de Louis XIV}

L'Eau qui danse... est une œuvre de la maturité de Gilles Tremblay, comme c'est aussi le cas des Piacevoli Notti de Giovan Francesco Straparola (Waters, 2001, p. 871). En effet, le conte Ancilotto, roi de Provino est la sources principale du conte pastoralo-aristocratique de Madame d'Aulnoy La Princesse Belle-Étoile et le Prince Chérot publié en 1698 dans le recueil des Contes nouveaux ou les fées à la mode, qui fonde le livret de l'opéra. Ce mystérieux auteur italien de la Renaissance aurait lui-même puisé aux sources orales de la Méditerranée et des Mille et une nuits ${ }^{6}$, puisqu'on peut rapprocher Princesse Belle-Étoile du conte des Deux Sœurs fondé sur la jalousie féminine (Zipes, 2001, p. 220), qui inspira également Grimm pour Les Douze Frères.

Le bel esprit donné à la Princesse Belle-Étoile par Madame d'Aulnoy est une survivance de l'esprit des conteurs de salon, un monde aristocratique et mondain où la parole est au cœur de la vie, tissage de productions de l'imaginaire et de badinages sur le ton de la conversation naturelle (Jasmin, 2008, p. 14-15) et la rouerie de Feintise en est le double négatif. Madame d'Aulnoy fut l'une des plus prolifiques et brillantes conteuses du règne de Louis XIV (Velay-Vallantin, 1992, p. 33), et belle-mère du compositeur Henri MontanBerton7. Pierre Morency relève le défi de la réécriture, n’hésitant pas à enrichir son inspiration d'autres versions du conte, dont quelques-unes ont été recueillies au Québec (Tremblay, Morency, Bellefeuille, 2009, p. 5) tandis qu'un souci dramaturgique le conduit à simplifier l'action en synthétisant les intrigues parallèles.

Ainsi la généalogie de Belle-Étoile est-elle réduite à sa plus simple expression : la grand-mère des enfants est «la Belle Fricasseuse », nom particulièrement évocateur que ne renierait pas Claude Ponti ${ }^{8}$, et une fée sauvée par elle
5. Les Piacevoli Notti ont été traduits en français par Jean Louveau en 1560 et complétés par Pierre de La Rivey en 1585 .

6. Il est délicat d'établir un lien entre Madame d'Aulnoy et la traduction d'Antoine Galland puisqu'elle a paru entre 1704 et 1717 (Miquel, 2005, p. XxxVII-XXXVIII). Si les entreprises sont peu ou prou contemporaines, la principale source de la poétesse reste incontestablement Straparola.

7. Henri Montan-Berton (1767-1844) composa un opéra d'après Madame d'Aulnoy: Ponce de Leon, créé en 1797 à l'Opéra-comique.

8 Écrivain et illustrateur français né en 1948, auteur de contes publiés à Paris à L'École des loisirs comme L'Arbre sans fin (1992). 
9. Créé au festival d'Aldeburgh le 11 juin 1960, avec Alfred Deller dans le rôle d'Oberon. Le livret de Britten et Pears s'appuie sur la pièce de Shakespeare dont le merveilleux marqua profondément Olivier Messiaen (1971, p. 8). de l'inanition protège ses enfants. Des trois sœurs subsiste principalement Blondine, la mère des trois enfants merveilleux; Brunette a épousé le frère du Roi mais meurt en couches: "Quant au fils de Brunette, il a perdu sa maman à l'accouchement» (Morency, 2009, p. 5). La troisième sœur n'est évoquée qu'au premier tableau par un évasif et anonyme «À mes deux sœurs et moi $[\ldots]$ » (Morency, 2009, p. 3) qui ne laisse entendre aucune jalousie, éludant la problématique de la rivalité entre sœurs, fondatrice des versions antérieures des Mille et une nuits de Straparola et de Madame d'Aulnoy.

Enfin, la vie chez le couple de corsaires qui a recueilli les quatre enfants et que Madame d'Aulnoy développe en dix pages (d'Aulnoy, 2008, p. 382-391) est réduite à sa plus simple expression: au troisième tableau de la première partie, Yby rapporte un bref dialogue entre Corsaire et Corsine donnant quelques explications sur les conditions de découverte des enfants et montrant l'ouverture d'esprit des parents adoptifs, prêts à laisser partir les jeunes gens à la découverte de leur origine. Il est aussi mentionné que le navire affrété à cet effet, aussi riche que l'esquif du premier voyage était précaire, est construit «sur ordre de Corsaire» (Morency, 2009, p. 7).

En revanche, Morency imagine le rôle d'Yby, conteur-griot au nompalindrome formé en miroir de l'avant-dernière et de la seconde lettre de l'alphabet, dont la voix tantôt déclamée, tantôt chantée est soulignée par les mélopées rythmiques du tambour parleur. Ce personnage central est entièrement nouveau, mais pourtant sous-jacent chez Madame d'Aulnoy puisque le conte est mis en abyme du fait de sa narration par des amis lors d'une réunion, comme dans le modèle des Mille et une nuits ou encore celui des Piacevoli Notti. Toutefois, Yby est proche de l'Oberon du Songe d'une nuit d'été de Benjamin Britten', opéra en trois actes que l'on peut sans conteste qualifier de féérique. En effet, Yby apparaît nimbé de la musique des abeilles, huit violons parfois rehaussés de percussions, comme Oberon est entouré de son essaim magique constitué de harpe, clavecin, célesta et percussions. Mais il s'apparente aussi au personnage de Puck, rôle parlé accompagné de tambour et trompette.

Pierre Morency modernise le texte en l'épurant et en le transformant en dialogues, sans perdre la poésie de Madame d'Aulnoy, en particulier dans son rapport à la nature et aux «bestiolinettes» (d'Aulnoy, 2008, p. 375). Toutefois, il adopte un style kaléidoscopique donnant à la langue une portée imagée et symbolique en fonction du contexte dramatique. Ainsi, la jubilation syntaxique caractérise le personnage sinueux et séducteur de Feintise. À la manière d'un alchimiste, elle joue avec les contraires: 
En tout lieu de ce monde est-il une jeunesse

Qui ne flambe comme un soleil dans la fontaine?

L'envol du feu qui danse

C'est la beauté de l'esprit et du corps. (Morency, 2009, p. 10)

ou scande les allitérations lorsqu'elle décline ses qualités à Belle-Étoile en une formule dont le caractère maléfique est accentué par l'émission en Sprechgesang:

FIGURE 1 L'Eau qui danse... deuxième partie, premier tableau (Tremblay, 2010, p. 45)

\section{Vif et rythmé}

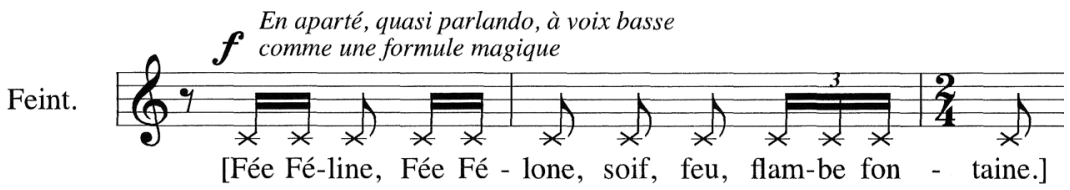

À ce discours trompeur répond à la fin de l'opéra-féerie l'énonciation poético-prophétique de l'Oiseau a capella sur un intervalle de quarte descendante: «Je ne veux pas dire, je dis» qui explique aussitôt:

En chantant je dénonce le mensonge de tous les temps.

En chantant je dénoue l'être des vivants

Et ainsi je promets de relier tous les êtres

Dans le poème enchanté où s'éclaire l'origine. (Morency, 2009, p. 29)

Enfin, si l'Oiseau est libéré dans le conte de Straparola et dans l'opéraféerie afin que Vérité puisse continuer à rimer avec Liberté, dans le conte de Madame d'Aulnoy il reste la possession des puissants et des sages, enfermé dans « une petite cage d'or d'un travail merveilleux» (d'Aulnoy, 2008, p. 428). Dans l'imaginaire des artistes, la question de l'absolutisme domine sous Louis XIV tandis que l'espoir habite la Renaissance et le début du XXI siècle.

\section{L'Eau qui danse, la Pomme qui chante et l'Oiseau qui dit la vérité: argument}

L'action est présentée par un narrateur, Yby, acteur et baryton, moitié oiseau, moitié abeille. Dans la symbolique de l'opéra cela signifie qu'il dit la vérité et qu'il accompagne les entrées et sorties des protagonistes. Il est suivi de son ombre musicale, son écho: un tambour-parleur qui ponctue ou commente son discours. Yby introduit chacun des dix-huit tableaux sauf le premier et le dernier par la formule «Il y eut» et après quelques phrases de 
contextualisation - lieu, action, personnages -, il laisse se dérouler l'action, intervenant ou non en sa qualité de conteur.

FIGURE 2 Jean Maheux dans le rôle d'Yby, L'Eau qui danse..., Montréal, Chants Libres 2009, mise en scène de Robert Bellefeuille, costumes de Marianne Thériault. Photo: Mathieu Dupuis.

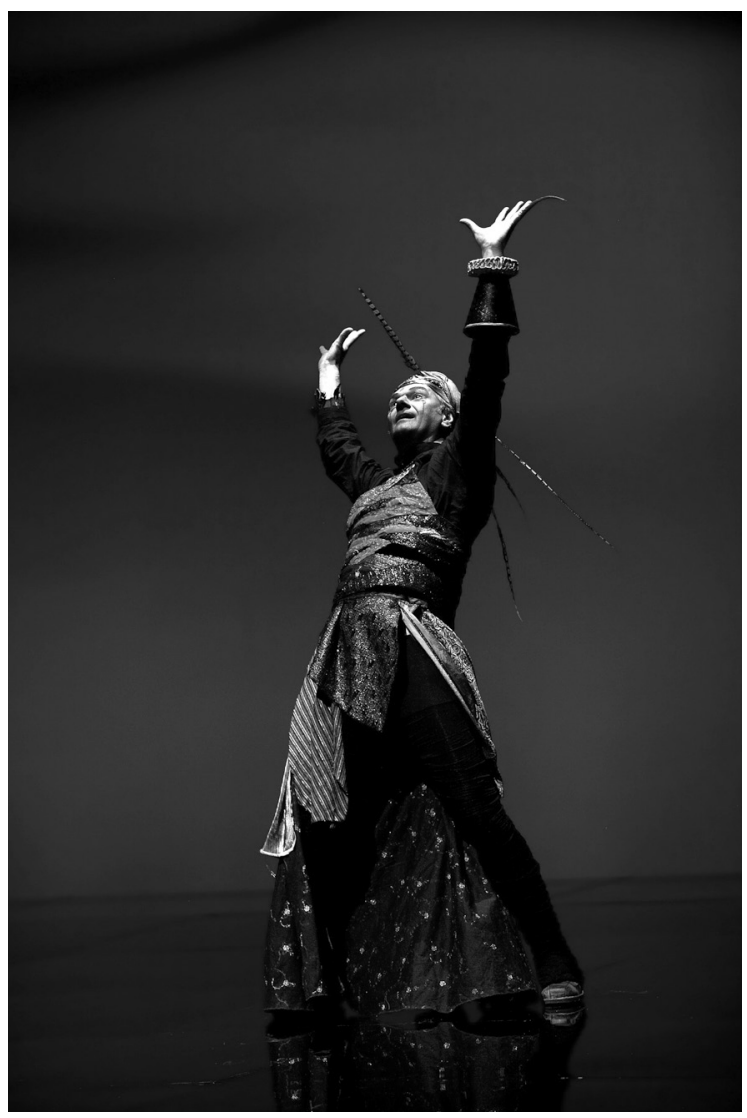
ont le cou ceint d'une chaîne d'or que nul ne peut enlever et de leurs cheveux s'échappent de subtiles pierreries.

Il s'agit d'une quête de soi à travers celle des origines, enchâssée dans un complot ourdi par la jalousie féminine.

Première partie. Le Roi a épousé une belle inconnue qui lui a promis de lui donner trois enfants (premier tableau). Furieuse, la Reine mère Poulane décide, avec l'aide de sa suivante Feintise, d'échanger les trois enfants merveilleux ${ }^{10}$ qui viennent de naître de cette union avec trois chiots et de tuer les enfants (deuxième tableau). Leur est associé dans ce noir dessein leur 
cousin, dont la mère, sœur de la jeune femme du Roi, est morte à sa naissance. Feintise, incapable d'assassiner elle-même les enfants, les dépose dans un frêle esquif sur la mer démontée. Or, protégés par la fée que leur grandmère avait nourrie et sauvée, ils sont recueillis par un Corsaire qui les confie à sa femme : tous deux les élèvent comme leurs propres enfants (troisième tableau). Arrivés à la fin de l'adolescence, une attirance irrésistible pousse Belle-Étoile vers Chérot son cousin et lorsqu'elle découvre au détour d'une conversation de ses parents adoptifs qu'ils ne sont peut-être pas frère et sœur, elle décide de les mener en quête de leurs origines, sous la protection avérée des corsaires et la garde de la fée (quatrième tableau). Revenus dans leur pays natal (cinquième tableau), les jeunes gens rencontrent le Roi. Ébloui par leur beauté, il se confie à sa mère qui comprend la trahison de Feintise : la suivante assure qu'elle mûrit un nouveau plan (sixième tableau).

Deuxième partie. En flattant Belle-Étoile, Feintise lui conseille, pour conserver une éternelle jeunesse, d'envoyer l'un de ses frères chercher l'Eau qui danse. Chérot accepte (premier tableau). Aidé par une tourterelle qu'il avait jadis sauvée, incarnation de la fée protectrice ${ }^{\text {11 }}$, il réussit à vaincre les sortilèges de la forêt et à rapporter l'Eau qui danse à Belle-Étoile (deuxième tableau). Apprenant le retour de Chérot, Feintise répond à la colère de Poulane que ce n'est que le début de son plan (troisième tableau). Elle revient vers Belle-Étoile et la convainc de demander à l'un de ses frères de lui cueillir la Pomme qui chante et donne de l'esprit (quatrième tableau). Dans un monologue, la jeune fille exprime son dilemme: «Je ne veux pas, mais je veux sans vouloir et je refuse tout en désirant» (Morency, 2009, p. 16) (cinquième tableau). Dans sa quête, Chérot rencontre un vieil homme qui lui indique le chemin de la Pomme; la tourterelle le rejoint et lui procure une armure faite de nombreux petits miroirs afin d'effrayer le monstre, gardien du fruit magique: il la cueille (sixième tableau). Yby conte le retour du Prince (septième tableau); Feintise enrage et informe Belle-Étoile de l'Oiseau qui dit la vérité, le seul moyen pour elle de connaître le mystère de sa naissance; voyant le désarroi de sa sœur, Chérot part à la recherche de l'Oiseau (huitième tableau). Mais arrivé près de l'Oiseau, Chérot est pétrifié: «Difficile est le chemin vers le vrai de la vie./ Celui qui monte sans savoir vers quel chant il s'élève/Celui-là ne peut entendre que la musique d'en bas » (Morency, 2009, p. 24): les deux frères de Belle-Étoile, partis à sa recherche, connaissent le même sort (neuvième tableau). Plongée dans la détresse, elle part à son tour et rencontre la tourterelle qui l'aide à capturer l'Oiseau : il lui enseigne comment redonner vie à ses frères (dixième tableau).
11. Et aussi de l'écrivain-librettiste-poète si l'on en juge par cette injonction à Belle-Étoile: "chante le meilleur de ta musique et laisse-moi faire le reste" (Morency, 2009, p. 26). 
Troisième partie. Yby conte la ruse de Poulane, qui, croyant les enfants morts dans les glaces sur les dires de Feintise, a convaincu son fils de se remarier (premier tableau). Mais le mariage est bouleversé par l'arrivée des enfants avec leurs trésors. L'Oiseau révèle la vérité sur leur naissance et confond les traîtresses. Chérot et Belle-Étoile sont réunis, le Roi rend sa liberté à l'Oiseau et Yby conclut à l'atemporalité de ce conte (second tableau). La vie reprend son cours [épilogue].

\section{Une métamorphose dramaturgique}

Les trois parties de l'œuvre suivent la tripartition classique exposition/péripétie/dénouement. Le temps dramaturgique est quant à lui divisé en deux moments distincts, suivant le principe d'encadrement narratif. Le premier et le dernier tableau sont déclinés au présent autour de la joie du Roi chantée par les abeilles au début, par l'Oiseau qui dit la vérité à la fin. Les autres, presque toujours introduits par la formule «Il y eut» sont placés dans le passé de narration et se succèdent en une mosaïque d'actions distinctes de dimensions inégales et impliquant presque toujours un changement de lieu, suivant le modèle des scènes du Wozzeck de Berg, chacune requérant un ou plusieurs styles, appropriés au contenu poético-dramatique.

Car l'étude de la mise en musique des contes de fées montre comment la «fiction avouée » (Biget-Mainfroy, 2001, p. 256) du conte permet le développement d'expérimentations musicales et dramaturgiques sous couvert de caractérisation de l'«Ailleurs intemporel » (ibid., p. 242) et favorise «une esthétique des mélanges» (ibid., p. 259). Ainsi, pour transformer le conte de fées de Madame d'Aulnoy en opéra-féerie, les artistes ont exploité en scènes closes, comprises comme des illustrations du récit d'Yby presque à la manière des flash-back cinématographiques, quelques éléments fondateurs du conte: la jalousie féminine, l'abandon, la bienveillance, la quête, la rédemption par le courage, l'amour, l'honneur et la mansuétude des puissants à l'égard des faibles.

Le vaste sommaire précédant les partitions de l'œuvre (Tremblay, 2007, p. 9; 2010, p. ii-xii) reprend non seulement le numéro des pages (deuxième colonne de chiffres) mais aussi le repère sur la partition (première colonne). Il est conçu comme un descriptif à la fois théâtral et musical de l'œuvre, suivant le modèle du traitement cinématographique, donnant pour chaque tableau un découpage interne en phrases résumant le contenu dramatique mais aussi musical et scénographique de chaque séquence, comme le montre l'exemple suivant: 


\section{Neuvième tableau.}

Yby, tambour parleur, l'Oiseau, Chérot, Chérot acrobate, Beaujour, Petit soleil.

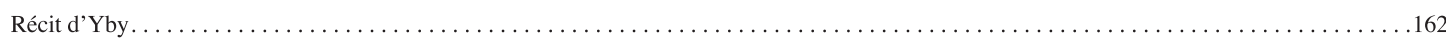

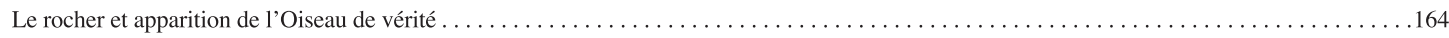

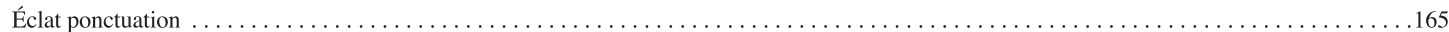

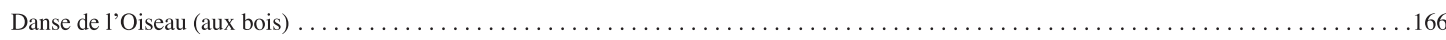

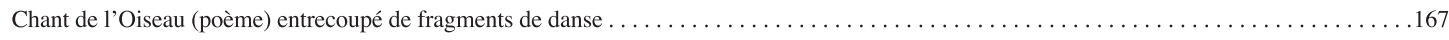

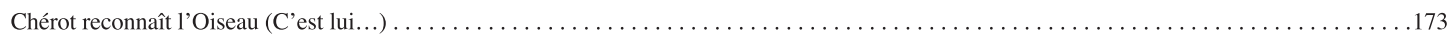

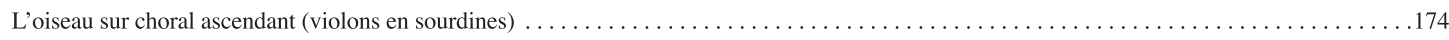

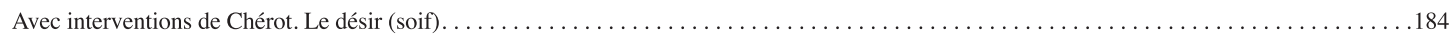

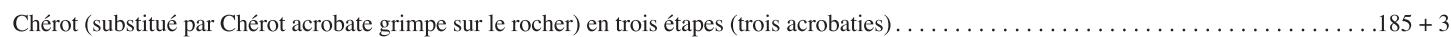

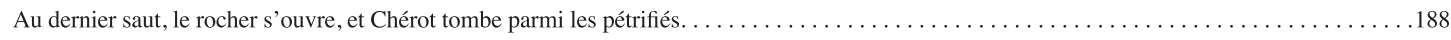

Seuls les yeux bougent et communiquent. (Projections d'yeux stylisés sur les murs dans la salle. . . . . . . . . . . . . . . . . . .

Poème de l'Oiseau (Difficile est le cheminement) avec ponctuations des claviers. . . . . . . . . . . . . . . . . . . . . . . . . . .

Transe de l'Oiseau et éclat, fondu enchaîné de la clarinette (sert de « conduit» à l'arrivée des deux frères) $\ldots \ldots \ldots \ldots \ldots \ldots \ldots \ldots \ldots \ldots$

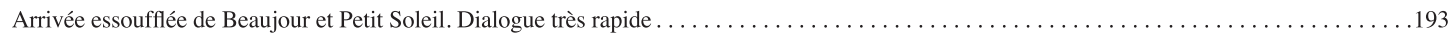

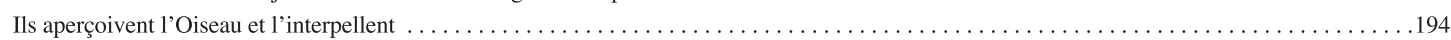

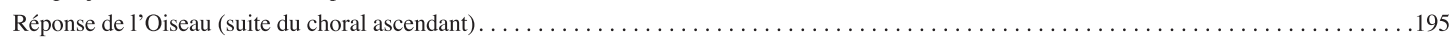

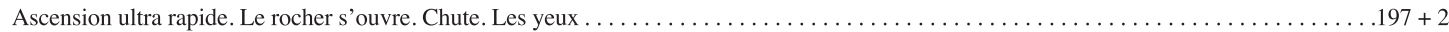

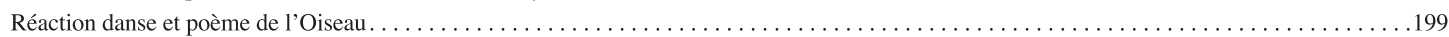

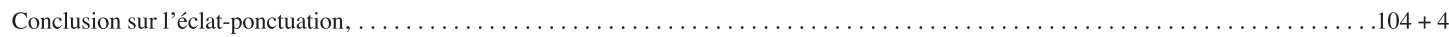

Dont la résonance est prolongée par les voix (hors scène), et le «HUM» amplifié et spatial de la cymbale. NOIR ...................205

Car la conception de l'œuvre est d'une rigueur millimétrique : sur les partitions figurent non seulement les textes parlés, chantés, la didascalie et la musique, mais aussi de nombreuses indications de mise en scène, décor, effets spéciaux, chorégraphie, et même d'acrobatie ${ }^{12}$.

Le déroulement dramatique alterne des récits faisant avancer l'action unifiés par la présence du conteur accompagné du tambour parleur, des scènes chantées, rythmées ou déclamées conçues comme des miniatures ou des séquences filmiques bien circonscrites et des intermèdes orchestraux chorégraphiés ou mimés permettant des suspensions dramatiques. Lors des commentaires du tambour-parleur et dans les interludes orchestraux les spectateurs peuvent cheminer intérieurement et appréhender la dimension métaphysique du conte.

Dans la construction en arche posée sur les piliers des parties extrêmes est développé, dans la deuxième partie, un jeu de contrastes entre le bien et le mal, succession de moments d'accalmie (retour de Chérot) et de désir, d'attente et de péripétie reliés les uns aux autres par la parole. Ainsi, fondant la dramaturgie, le désir est instillé dans le cœur de Belle-Étoile par la parole de Feintise qui manque néanmoins sa cible: «Tout ce que j’entreprends se retourne contre moi / Alors que la plupart réussissent à faire mal en voulant 
13. Morency n'évoque pas leur sort, pourtant clair dans les versions antérieures: la mort, la réclusion ou le bannissement. Un geste du Roi montre, dans la mise en scène de la création, que Feintise et Poulane sont bannies. Le son des "arco-grains" laisse penser que Gilles Tremblay leur souhaite de voir rejoindre le mur d'ossements (Tremblay, 2007, p. 296).
14. Même si cet élément est le moins prégnant à l'opéra mais se retrouve dans les personnages de faire valoir qui émaillent les œuvres comme le couple Papageno/Papagena de La Flûte enchantée ou les frères de Barak dans La Femme sans ombre.

15. Dans son Vocabulaire de la langue thêatrale, Alfred Bouchard (1878, p. 116) définit la féerie comme une "pièce à grand spectacle, où le sujet est remplacé par les décors, le dialogue par les trucs, l'esprit par des actrices deminues, quand elles ne le sont pas tout à fait, au maillot près". faire le bien / Je n'arrive qu'à faire du bien en manœuvrant d'horribles actions» (Morency, 2009, p. 20).

Répondant au banquet des noces du Roi et de Blondine qui fermait la première partie, la noce finale du Roi et de la nouvelle épouse est le lieu du dénouement, de la révélation, de la métamorphose, suivant le principe de fête dévastée, archétype dramaturgique de l'opéra (Auzolle, 1995). Chacun quitte son masque et devient qui il est profondément. La dévastation a lieu dans la mesure où la méchanceté des antagonistes est reconnue ${ }^{13}$, mais la fête, comme la vie, continue. Dans le conte, le rideau tombe sur la liesse générale à l'inverse de ce qui avait présidé à la mise en place de la dramaturgie: alors que la cour aurait dû se réjouir de la naissance des princes, les antagonistes ont ajourné cette joie par leur funeste projet qui a néanmoins permis à la noblesse de cœur des protagonistes de se révéler. Ils peuvent alors accéder à leur rang non uniquement par la naissance mais aussi par le mérite: le pouvoir ne s'acquiert pas par filiation mais par initiation. À l'époque de Madame d'Aulnoy comme aujourd'hui, cette allégorie de la nécessité de l'accomplissement de hauts faits pour revendiquer une quelconque légitimité revêt une indubitable résonance politique.

L’une des spécificités de l'opéra français baroque à l'âge de la tragédie classique est, en vertu de son rôle événementiel, de pousser la musique du côté de la langue (le théâtre), du mouvement (la danse) et du spectacle (Kinztler, 2002). Car l'opéra français du tournant des XVII ${ }^{\mathrm{e}}$ et XVIII ${ }^{\mathrm{e}}$ siècles montre ce que cache la tragédie classique: il exprime le non-dit grâce à la dimension musicale, phénomène plus tard maîtrisé et utilisé par Wagner. Aussi, lorsqu'il met en musique un texte venu de ce temps de la retenue et de la magie, comment Gilles Tremblay s'inscrit-il dans une tradition tout en lui insufflant sa spécificité?

\section{De la féerie à l'opéra}

L'Eau qui danse... répond aux cinq critères définis par Jean-Claude Yon (2006, p. 126-133) comme constitutifs de la féerie telle que déclinée par les artistes du Second Empire, mais qui, peu ou prou, caractérise toute œuvre lyrique empreinte de merveilleux depuis Lully. Il s'agit de la présence de merveilleux mêlé d'aventures, du comique verbal'14 conçu «[...] comme contrepoint à l'éblouissement visuel » (ibid., p. 130), de l'éclat des décors et des mouvements scéniques, de l'abondance des transformations et du rôle essentiel dévolu à la musique. Les féeries ${ }^{15}$ répondent avant tout à la nécessité d'éblouir, d'émerveiller dans une surenchère d'effets et de contrastes; 
inversement, l'œuvre de Gilles Tremblay et Pierre Morency cherche à atteindre une forme d'épure autorisant à sortir du Regietheater pour retourner vers le rêve et la poésie.

Le caractère composite du conte de fées souligné par les historiens et cultivé par Pierre Morency est estompé ici par la composition musicale et la scénographie: si Mozart a multiplié les ruptures de ton et de style dans La Flûte enchantée, Strauss a inventé une musique complexe servie par une orchestration à la fois foisonnante et subtile destinée à souligner le caractère merveilleux du livret d'Hofmannsthal pour La Femme sans ombre. Gilles Tremblay propose quant à lui un discours musical homogène, avant tout ancré dans son propre style nourri de multiples influences par exemple la monodie médiévale, les sons de la nature, la matière sonore varésienne, les jeux de gongs du théâtre chinois, le gamelan balinais et s'enrichit, pour cette œuvre de celle de l'Afrique de l'Ouest et centrale (Owomoyela, 1993) à travers l'utilisation du tambour parleur ${ }^{16}$, considéré comme un instrument divin, donc symbolique dans le contexte de cet opéra-féerie.

Gilles Tremblay épouse la construction en arche du livret en structurant le temps de l'œuvre grâce à des repères forts: le rôle récurrent du conteur, le motif de la marche qui permet d'entrer dans le palais du Roi ${ }^{17}$, la musique magique des abeilles ${ }^{18}$, le violoncelle plaintif de Feintise, la trompettecorneille de Poulane, le cor de Tourterelle ou les «arco-grains» des trépassés. Chaque tableau réserve de nouvelles couleurs instrumentales, vocales, pour le plaisir et l'émerveillement du public et dans l'authenticité du style de Gilles Tremblay. Car L'Eau qui danse... n'est pas un électron libre : c'est l'opéra d'un compositeur dans toute la possession de ses moyens, le fruit d'un mûrissement non seulement sur le sens intrinsèque de l'œuvre musicale mais aussi sur son rapport au monde dans lequel elle advient.

Une analyse détaillée de cet opéra-féerie risquerait d'en rompre le pouvoir d'enchantement, pour reprendre un terme que Bettelheim (1976, p. 36) emprunte à La Bruyère. Car le charme puissant de cette œuvre, souligné par tous les commentateurs de la création, demeure une fois le rideau tombé. Toutefois, un analyste traquant dans L'Eau qui danse... la trace des préceptes compositionnels d'Olivier Messiaen en termes de rythmes, de modes et de chants d'oiseaux sera vite comblé, comme celui qui cherchera les racines de cet opéra dans l'œuvre antérieure de Gilles Tremblay. Quelques critères stylistiques prégnants, tant sur le plan vocal qu'instrumental, peuvent néanmoins être déclinés.
16. vivrelemonde.free.fr/ instrumentarium.php

17. Voir figure 9.

18. Voir figure 11. 
19. Les références dans les partitions (Tremblay, 2007 et 2010) sont désormais abrégées: (1, 1:24).
20. Poulane est sans doute le rôle dont la voix est la plus malmenée puisque la chanteuse halète, suffoque, tousse, émet des "cris terribles et rauques" (Tremblay, 2007, p. 11), jeu renforcé par son ombre instrumentale, une trompette imitant une "corneille enragée" (ibid., p. 27).

\section{Du texte au chant: la vocalité}

L’opéra-féerie comporte douze chanteurs, huit relevant d'une nomenclature traditionnelle, quatre de registres plus atypiques. Les rôles d'ingénue (Belle-Étoile) et de femme noble (Blondine) sont confiés à des sopranos légers, tandis que les traîtresses ont des voix graves (Poulane, alto; Feintise, mezzo-soprano). Le Roi, noble et sage, est une jeune basse, utilisant pourtant sa voix de fausset lors de sa transe de désir ( $1^{\text {re }}$ partie, $1^{\text {er }}$ tableau: repère $24^{19}$ ); Chérot, l'amoureux héroïque est ténor; Petit Soleil et Beau Jour, ses acolytes, des barytons moyens. Les quatre êtres merveilleux sont caractérisés par des voix plus originales: la fée Tourterelle est contralto, la Pomme qui chante et la Sirène sont chantées par une mezzo-soprano hors scène, employant principalement des voyelles en vocalises, le narrateur Yby, autant conteur que chanteur, est baryton et enfin l'Oiseau est haute-contre. Les figurants ne chantent pas, il s'agit donc d'un opéra sans chœur, même si le tutti des solistes donne une seule et brève fois un effet choral $(3,2: 48)$ pour signifier la consternation générale devant l'ignominie de Poulane et Feintise.

Sans dresser le catalogue des types d'émission vocale utilisés par Gilles Tremblay dans L'Eau qui danse..., quatre principales techniques peuvent être distinguées: une déclamation s'apparentant au jeu de l'acteur, quasi exclusivement pour Yby, un parlé-rythmé plus ou moins proche du Sprechgesang mais prenant de très nombreuses nuances en fonction des personnages et des situations ${ }^{20}$, une monodie a capella oscillant entre le récitatif et l'arioso et enfin un geste vocal intrinsèquement lyrique, dans la tradition française Debussy-Poulenc-Messiaen. Jouant sur la multiplicité des émissions, il utilise l'ambitus total de chaque voix afin d'en varier les couleurs, même dans les airs ou les ensembles, balayant ainsi tout le spectre des comportements humains.

La richesse expressive de l'écriture vocale de Gilles Tremblay est d'autant plus mise en valeur qu'il l'enchâsse dans le tissu instrumental plutôt que l'accompagner, se situant à cet égard également dans la lignée de Debussy et de Messiaen. Cette conception infiniment respectueuse de l'instrumentvoix apparaît dès Kekoba, où il explorait ses possibilités dans un esprit foisonnant caractéristique des années 1960, demandant par exemple au ténor d'employer sa voix de fausset, préfigurant l'Oiseau de L'Eau qui danse... Dans Kekoba aussi, il fait alterner la voix et les instruments, sans mélange, avec juste des superpositions de résonances à l'articulation des discours; seulement pour l'Amen les deux se rejoignent dans une apothéose sonore, un principe retrouvé dans Les Vêpres de la Vierge (1986) où les instruments 
utilisés en soli monodiques (la flûte en particulier) amènent les voix, chorales ou solistes comme le «Magnificat» final pour soprano a capella vocalisant.

Cette écriture est largement utilisée dans L'Eau qui danse..., puisque la voix chantée se fait presque toujours entendre en monodie a capella ou sur des résonances comme lors de la promesse de Blondine (1, 1: 16-19), du "Chant de l'Oiseau » (2, 9:167-173) ou du «dernier poème de l'Oiseau » (3, 2: 76-89), la voix de haute-contre étant utilisée «dans une liberté désinvolte et fantaisiste» (Tremblay, 2007, p. 206).

Les séquences unissant un groupe instrumental et des voix sont exceptionnelles et destinées à conférer emphase ou lyrisme au chant dans les moments d'exultation ou de séduction. Dans ces passages - parfois très brefs - évoquant les grands airs du répertoire, la voix est toujours projetée dans la tradition lyrique, les intervalles des lignes mélodiques précisément indiqués. Il s'agit de l'évocation des cheveux magiques des enfants (1, 1: 19-20), du désir entre le Roi et Blondine (1, 1: 19-26), du bonheur de Corsaire et Corsine quand les enfants arrivent à leur foyer $(1,3: 73-75)$, du ravissement du Roi devant la beauté des enfants $(1,6: 125)$, de la séduction de Feintise envers Belle-Étoile (2, 1: 4-6; 2, 8: 129-142), des pensées de Belle-Étoile pour Chérot $(2,1: 10-14 ; 2,5: 72)$, de l'exaltation de Chérot pensant à Belle-Étoile colorée par une mixture d'harmoniques de clarinette, flûte et piccolo $(2,2: 50)$, des «bulles des amoureux » avant le départ de Chérot vers l'Oiseau (2, 8: 150-153) et au moment de la révélation finale de l'identité (3,2:58-65), des prophéties de l'Oiseau (2, 9: 174-183, 195-196; 2, 10:249; 3, 2: 49-50) et du chant de la Pomme (3, 2: 30-38).

La voix en "parlé-modulé-rythmé » (Tremblay, 2007, p. 8) est utilisée à nu, en dialogue avec un instrument (le tambour parleur et Yby) ou superposée à des alliances instrumentales incluant souvent les percussions pour leur impact rythmique et/ou pour leur timbre comme dans les imprécations de Poulane (1, 2:54; 1, 6: 136), ses dialogues avec Feintise (2, 3:53-59) ou encore dans un but descriptif comme la séquence des violons au jeu «arco-grains ${ }^{21}$ » $(1,6: 104-107 ; 3,2: 46)$.

Les différentes formes de vocalité sont réunies dans la scène finale $(3,2)$ où le Roi chante en arioso, l'Oiseau en arioso et en parlé, les autres protagonistes utilisant les voyelles rythmiquement (exclamations) ou mélodiquement (vocalises), jusqu'à maquiller le timbre en le rapprochant de celui des ondes Martenot piano dans le medium, comme Gilles Tremblay l'avait déjà expérimenté dans Oralleluiants. Feintise et Poulane n’ont alors plus « voix au chapitre » et sont remplacées par leurs ombres instrumentales: le violoncelle et la trompette.
21. "Les notes indiquées ne sont pas des hauteurs, mais des doigtés seulement colorant le bruit des grains", explique le compositeur, afin de caractériser le mur d'ossements que Chérot doit traverser pour atteindre la Pomme, interprétation contemporaine de la danse macabre, s'il en est; cf. aussi la note 12 dans l'article de Vincent Ranallo dans ce numéro. 
Si le théâtre, parlé ou musical, a considérablement nourri Gilles Tremblay, l'ancrage de L'Eau qui danse... dans la tradition est manifeste non seulement par le très large emploi de la vocalité lyrique dans l'expression de toutes ses nuances, mais aussi par le tissage des instruments avec les voix, d'une infinie richesse combinatoire.

\section{L'instrument: couleur, rythme et voix}

Rarement utilisé en tutti sinon dans les interludes ou les chorégraphies, l'orchestre est composé de vingt-cinq instrumentistes: piccolo, flûte, hautbois, clarinette en si bémol, basson, cor en $f a$, trompette en $u t$, trombones ténor et basse, piano, huit violons, violoncelle, trois contrebasses et trois percussionnistes jouant respectivement le glockenspiel, le xylorimba, la cymbale antique, trois cymbales turques (la grave étant amplifiée par deux micros mobiles), le grand shaï (wind gong) et cinq gongs accordés, le tambour parleur, trois bongos, deux congas, la caisse claire avec timbre et la caisse claire grave, le tambour à corde (lion's roar), les cymbales de bois et les maracas (premier percussionniste); le vibraphone, le xylorimba, cinq cymbales turques, deux gongs accordés, trois gongs de temple, deux tam-tams (amplifiés par deux micros), deux petits gongs de théâtre chinois à glissando ascendant, les grelots, la caisse claire avec timbre, le wood block, les maracas, le grand guero [sic] (deuxième percussionniste); le glockenspiel, les cloches-tubes, trois cymbales turques, la cymbale antique, deux gongs accordés, deux tam-tams graves, trois tom-toms, une grosse caisse très profonde, cinq wood blocks, deux maracas et une grande assiette d'aluminium (ou grande feuille) amplifiée par deux micros fixes (troisième percussionniste).

Les percussions dominent ainsi non seulement la palette sonore de L'Eau qui danse..., quasiment toujours présentes dans les associations timbriques, mais participent de la scénographie puisqu'elles sont réparties en deux groupes placés de chaque côté de la scène, une contrainte transcendée par Robert Bellefeuille qui commande à Marianne Thériault des costumes reprenant les tons noir, or et cuivre afin que les corps des protagonistes offrent un écho visuel au scintillement des percussions (Tremblay, Morency, Bellefeuille, Vaillancourt, 2010, «making off»).

Plusieurs instruments ou groupes d'instruments caractérisent un personnage ou une situation. En ce qui concerne les ombres instrumentales, la sonorité élastique du tambour-parleur accompagne le personnage d'Yby à chacune de ses interventions dans un effet de redondance (Carrington, 1971) et de soulignement, le violoncelle plaintif s'accorde à la fourberie de Feintise dans ses échanges avec la Reine mère $(1,1: 44-45,55-61 ; 1,6: 133-139$; 
3, 2: 71-72), imité par le tambour-parleur $(3,1: 6)$, la trompette-corneille à la méchanceté de Poulane (1, 1: 38-43, 46-49; 1, 6: 120-122, 130-132; 3, 2: 69-70). Quant au cor, il offre un écho à la voix profonde de la fée Tourterelle et lorsqu'il se fait entendre seul, le spectateur comprend que l'action est placée sous sa protection, comme dans la scène de tempête $(1,3: 64-69)$. La famille royale est définie par un motif de révérence et d'interrogation partagé entre le hautbois et la trompette $(1,2 ; 1,5 ; 2,3 ; 3,2)$ tandis que les huit violons en tutti symbolisent les abeilles magiques tout au long de l'œuvre. Enfin, le son «arco-grain» des huit violons représente le mur d'ossements des chevaliers aventureux (2, 6: 104-107) et induira l'idée de la mort des traîtresses (3, 2: 46).

Dans son évocation de la nature, Gilles Tremblay ne cède pas aux attendus de la musique descriptive, comme l'emploi de trilles et traits virtuoses pour camper l'Oiseau (L'Oiseau de feu de Stravinsky, Pierre et le loup de Prokofiev...) : pour le charmer, Gilles Tremblay unit brièvement la voix de Belle-Étoile à celle de la flûte, comme une réminiscence de l'appeau, mais le procédé est vite abandonné (2, 10: 219-220). Il s'agit d'une manœuvre de séduction, un leurre: l'Oiseau n'est pas une flûte.

FIGURE 4 L'Eau qui danse..., deuxième partie, dixième tableau (Tremblay, 2009, p. 111)
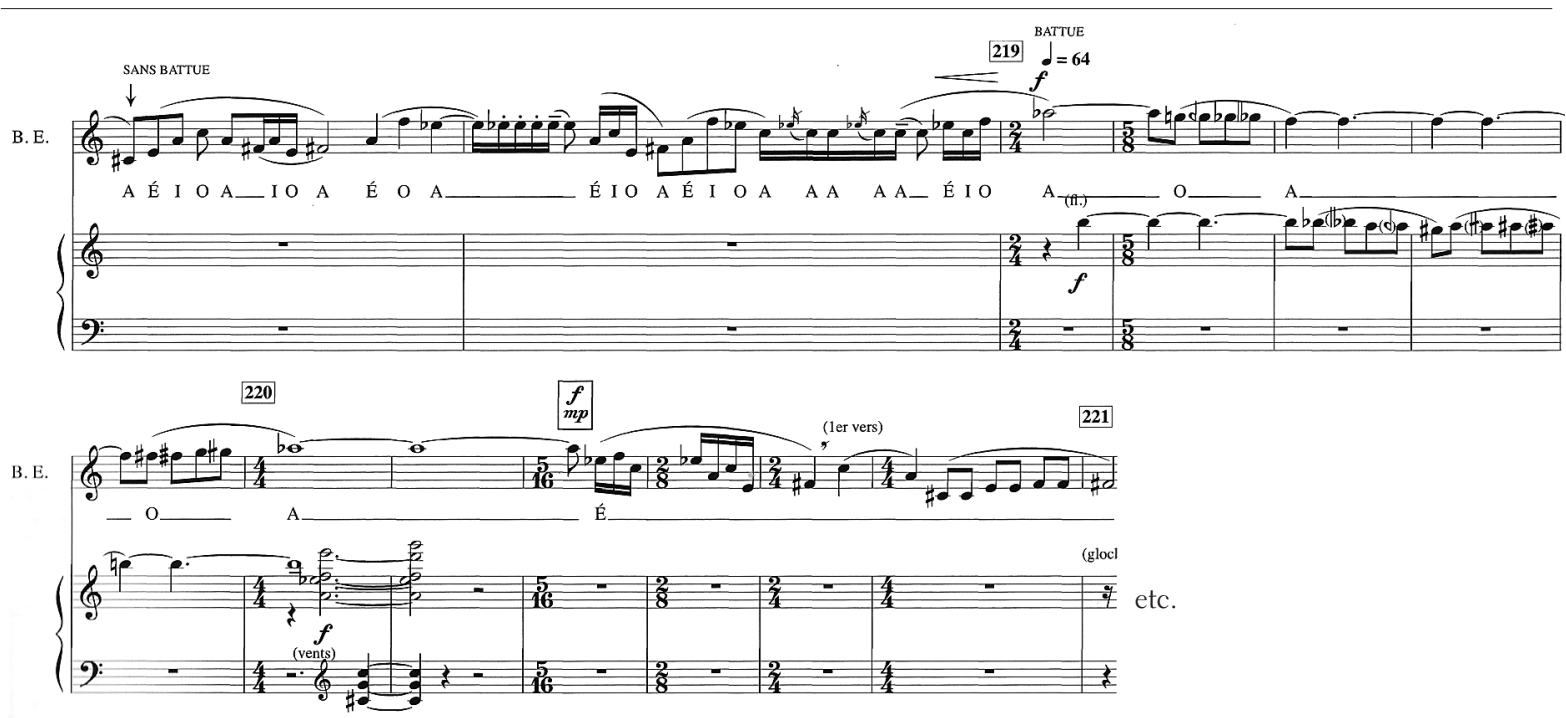

De même pour la musique de la tempête, chaque instrumentiste doit faire appel à son propre imaginaire pour interpréter les «pluies de notes », «rafales» ou «éclaboussures» $(1,3: 64-70)$. 
22. $35 \mathrm{~mm}$ et $16 \mathrm{~mm}$, couleur, 4 minutes 46 secondes; film sonore sans commentaire.

23. www.smcq.qc.ca/smcq/fr/artistes/t/ tremblay_gi/
Le compositeur avait déjà envisagé la question des rapports entre l'image et la musique pour le film Dimension soleils ${ }^{22}$ et remarquait « $[\ldots]$ une fois les premières associations images-sons établies, la musique, enrichie en cours d'aventure, chemine en sa propre dimension » (Tremblay, 1970). Même s'il s'agit là d'une démarche abstraite puisque le film ne comportait nulle action au sens théâtral du terme, les principes compositionnels et les symboles de L'Eau qui danse... sont en place : l'étoile, le soleil, le combat, la lumière, l'affrontement, dans une écriture en blocs de taches sonores, faisceaux d'harmoniques, constellations de hauteurs pensées en ombres et lumières, émergence et simultanéité, chatoiements et résonances. Les innombrables indications de jeu musical ou scénique portées par Gilles Tremblay sur sa partition montrent qu'il est impossible de dissocier les dimensions musicale et spectaculaire dans L'Eau qui danse... La féerie est conçue dès l'élaboration musicale par un compositeur ancré dans l'expression du merveilleux.

\section{Pour une esthétique de l'émerveillement}

Son ami Pierre Morency, se rappelant la conception de L'Eau qui danse..., rapporte l'enthousiasme de Gilles Tremblay: «Le réel est enchantement. Que s'enclenchent les féeries! L'Oiseau de vérité dissipe l'illusion au profit du merveilleux» (Tremblay, Morency, Bellefeuille, 2009, p. 5). La mère du compositeur écrivait des livres pour enfants ${ }^{23}$ et lui-même, aîné de cinq enfants, est également père et grand-père; aussi le conte et son pouvoir d'enchantement sont-ils au cœur de son rapport au monde, situant sa démarche à la frontière de la «magie» (ibid.) et de la poésie. Robert Bellefeuille est séduit par «l'effervescence » (Tremblay, Morency, Bellefeuille, Vaillancourt, 2010, «making off») de celui qui se définit lui-même (Tremblay, 1995, p. 61) comme:

\section{"Celui qui s'émerveille"}

Car Gilles Tremblay sait effectuer «la transcription d'une écorce de bouleau » (ibid.) pour constituer une partie du matériau de Compostelle I, cheminant d'un matériau visuel et tactile vers sa transposition musicale. Au-delà d'un simple choix de modèle sonore naturel, il montre un profond respect pour les événements de l'univers «Là où science et art se rejoignent en poésie, en contemplation» (ibid.), générant l'inspiration.

L'attrait pour la dimension merveilleuse de l'imaginaire déployée dans une acception mystique de l'univers préside à l'élaboration de Kekoba (1965) qui utilise successivement des "vibrations tournoyantes ", « un grand trait aux percussions, contrapunté par une "vocalise" des ondes» et finalement «des événements de plus en plus précieux et merveilleux» (Tremblay, Duchesnay, 1983, p. 8). La fréquentation du merveilleux permet de restaurer cette faculté 
d'émerveillement affirmée au contact d'Olivier Messiaen et déclinée au fil des années comme la «symbolique sonore d'une perception spirituelle du monde» (ibid., p. 5). Peut-être le surprenant succès des atypiques «Scènes franciscaines» de son maître, opéra de la maturité, chef-d'œuvre au sens compagnonnique du mot véritablement entré au répertoire des grandes scènes lyriques internationales, lui a-t-il donné le courage de composer à son tour un opéra qui, sans chercher à bousculer les conventions, renouvelle le genre tout en s'inscrivant dans la continuité de son histoire. Gilles Tremblay épouse et poursuit en cela la démarche du compositeur français dont l'attirance pour les contes de fées accompagna le développement de la foi catholique (Massin, 1989, p. 15) et qui affirmait: «Le Merveilleux est mon climat naturel, au sein duquel je me sens bien» (ibid., p. 27).

\section{Le merveilleux dans L'Eau qui danse...}

Le sous-titre donne le ton: «opéra-féerie » et il est manifeste que L'Eau qui danse... existe bien à la frontière des deux genres, réunissant les arts de la scène (chant, comédie, danse, mime, décor, effets spéciaux) et un orchestre traité de manière théâtrale, narrative et responsoriale, dans une esthétique participant de la féerie, de l'opéra chinois, du théâtre musical et de l'opéra. Le merveilleux tient pour une grande part à l'effet d'ombre ou d'écho offert par les instruments au discours chanté, reflétant les sentiments, les états d'âme des protagonistes dans une singulière dilatation du temps, mais aussi par la maîtrise des timbres et de leurs alliances, toujours traitées dans un souci d'économie de moyens puisque trois à cinq instruments est la combinaison la plus fréquente, telle que le trio marimba, xylophone et piano de la danse de l'Eau $(2,2: 39-52)$.

Sur la partition, chaque détail musical, mais aussi scénique, chaque effet sonore, chaque élément symbolique est imaginé, pensé, noté, prévu, expliqué, le cas échéant, dans un souci de méticulosité rare dans le domaine de l'opéra où les exigences scéniques viennent souvent bousculer l'imaginaire du compositeur ${ }^{24}$.

Pourtant, plus rien ne transparaît de cette horlogerie interne lors de la représentation car le temps spectaculaire n'est jamais saturé, les masses sonores sont dosées avec une grande rigueur, les voix, toujours compréhensibles, simplement rehaussées de touches orchestrales sans jamais de sentiment d'étouffement ou de pléonasme. Lors de la création montréalaise, dans cette scène, les tambours de basque ont été laissés de côté pour privilégier l'étrangeté du son des violoncelles sul ponticello mêlé à celui de la caisse claire, l'ensemble ad libitum.
24. On le voit en particulier dans les mises en scène qui ont succédé à celle de Saint François d'Assise organisée par Olivier Messiaen pour la création en 1983, notamment celle de Peter Sellars à Salzburg dès 1992. 


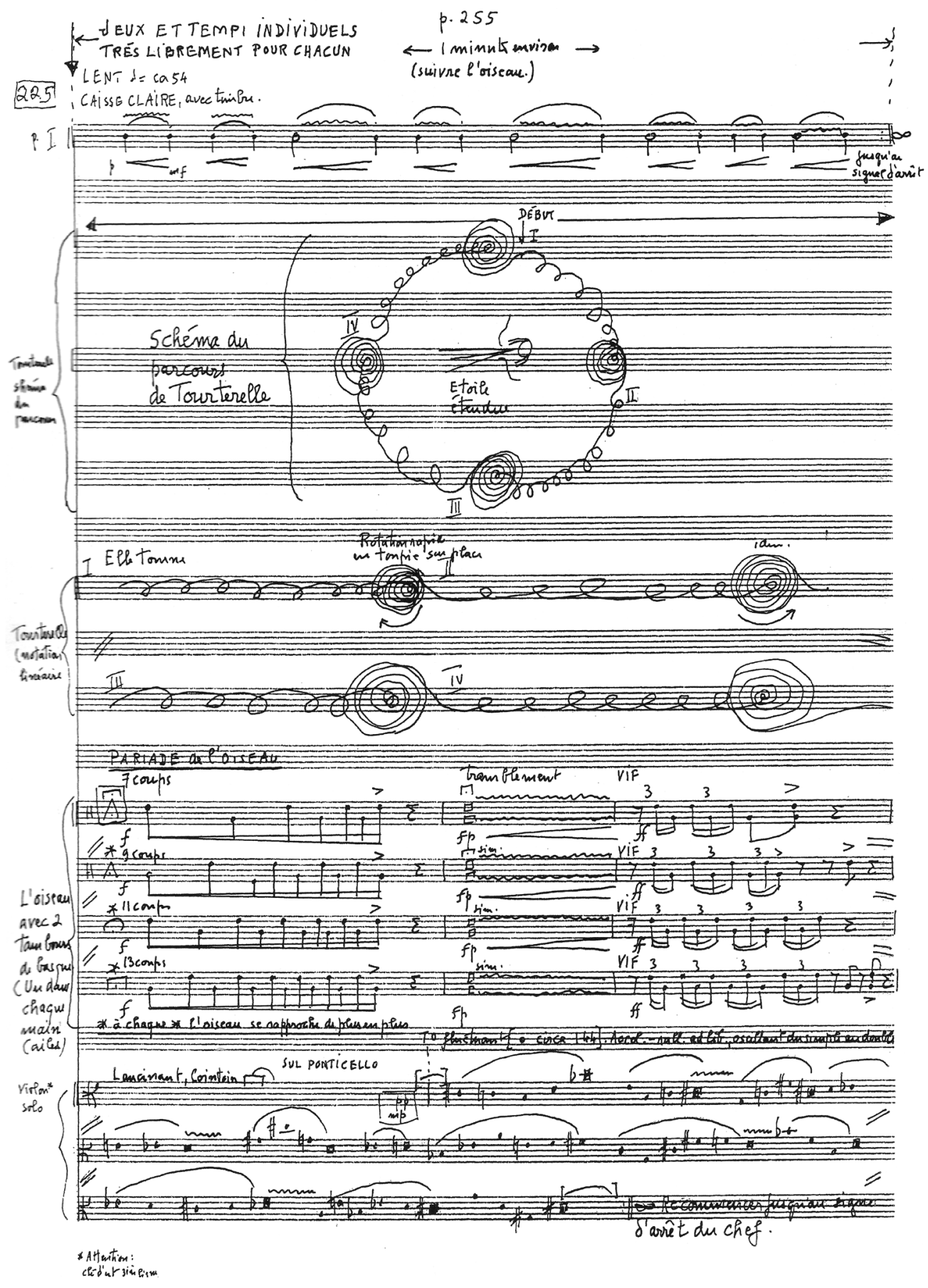


Quelques pages auparavant (2, 9: 214), le geste bienveillant de Belle-Étoile soufflant dans le bec de Tourterelle pour la ranimer est accompagné d'un son surnaturel, subtilement dosé par le compositeur:

FIGURE 6 L'Eau qui danse..., deuxième partie, neuvième tableau (Tremblay, 2007, p. 249)

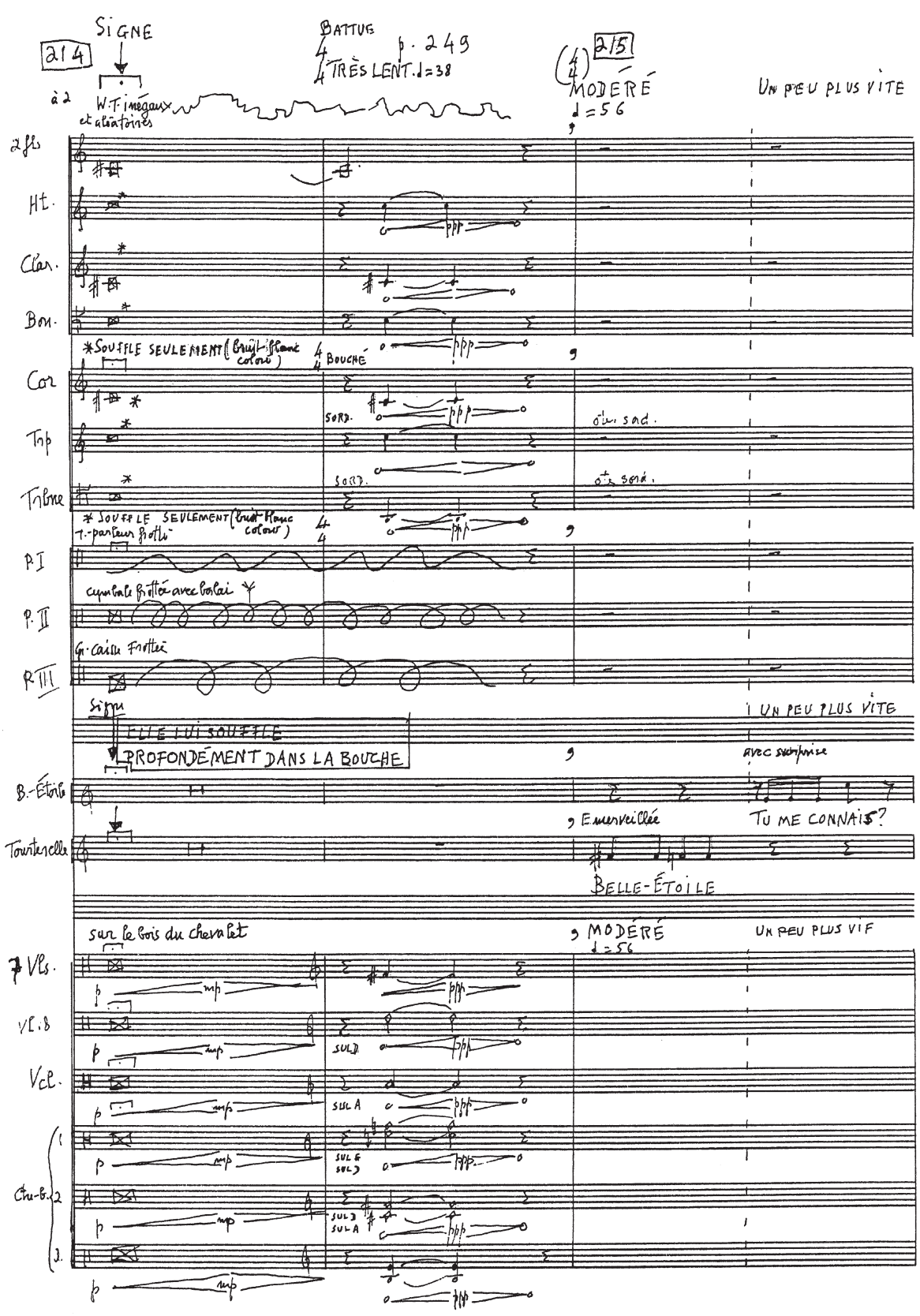


25. Comme celle d'Olivier Messiaen, en particulier dans Chants de terre et de ciel (1938), Visions de l'Amen (1943), Vingt regards sur l'Enfant-Jésus (1944), Harawi (1945), Turangalîla symphonie (1948), Cinq Rechants (1949), Et exspecto resurrectionem mortuorum (1965), Des Canyons aux étoiles (1974) ou de l'un de ses autres élèves, Karlheinz Stockhausen avec Sternklang (1971). Tremblay reprend le symbole de l'étoile dans Kekoba, (1965-1967), Compostelle I (1978) et Vers une étoile (Compostelle II, 1993); d'ailleurs, Kekoba signifie "étoile" en hébreu et on se souvient que l'Empereur de La Femme sans ombre de Strauss, figure tutélaire et guide de la quête de l'Impératrice de sa dimension humaine, se nomme Keikobad.

26. "Ainsi le mot "stella", comme une étoile, rayonne, éclate en plusieurs langues: latin, anglais, russe, bengali, chinois, français, arabe, hébreu " (Tremblay et Duchesnay, 1983, p. 8).

\section{7. "[...] le mot "étoile" correspond} [...] aux dispositions spécifiques des formants de base et par extension à l'idée de complémentarité qui s'y rattache" (Tremblay et Duchesnay, 1983, p. 17).

28. "[le mot "étoile"] évoque également l'Étoile des mages et toute la poésie de la liturgie de l'Épiphanie, notamment l'esprit de jaillissement en vocalises [...]" (ibid.).
Dans la posture caractéristique de Gilles Tremblay, le merveilleux qui nimbe le monde et auquel il suffit de prêter attention irrigue L'Eau qui danse... Feintise avoue ainsi à Belle-Étoile au début de la deuxième partie: «Le merveilleux marque toute votre personne à l'image de cette étoile d'or sur votre front» (Morency, 2009, p. 11). Or, l'étoile est un symbole qui parcourt l'œuvre de Gilles Tremblay'25, dans une pluralité de sens, éclatement linguistique ${ }^{26}$, acoustique ${ }^{27}$, mystique et poétique ${ }^{28}$. Trois des quatre protagonistes sont nés sous le signe de l'étoile dans l'exaucement féerique du vœu d'une jeune fille:

[mon vœu] est simple quoique extravagant: si un Roi veut m’épouser, je lui donnerai au bout de neuf mois deux beaux garçons et une fille magnifique, magnifique, magnifique. Si beaux qu'ils porteront au front une étoile d'or et au cou une chaîne étincelante. Leurs cheveux tomberont en anneaux et répandront les plus fines pierreries. (Morency, 2009, p. 3)

Une vocalise accompagne le mot «magnifique» $(1,1: 18)$ :

FIGURE 7 L'Eau qui danse..., première partie, premier tableau (Tremblay, 2010, p. 5)

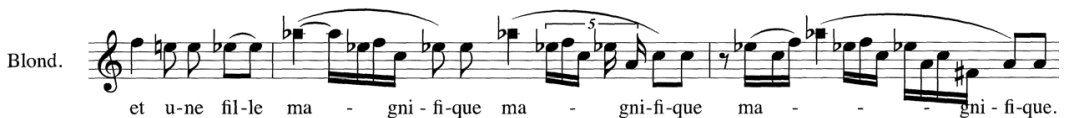

et l'alliance du glockenspiel, du vibraphone et du piano soulignent «étincelante» $(1,1: 19)$ :

FIGURE 8 L'Eau qui danse..., première partie, premier tableau (Tremblay, 2007, p. 14)

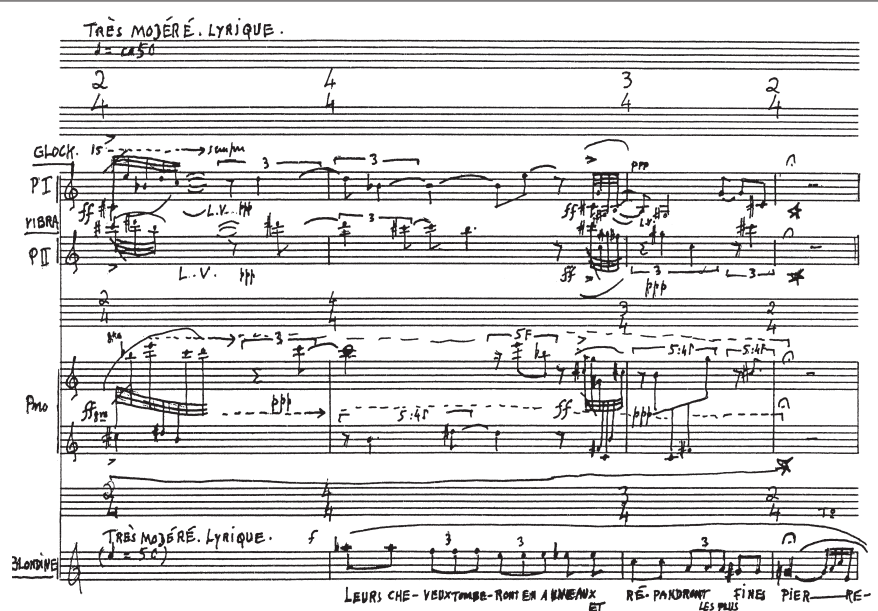


Au tableau suivant, la «ville merveilleuse » est symbolisée par un double motif: un ostinato mélodico-rythmique de tierce mineure joué sur deux gongs de temple et une mélopée trillée orientalisante au hautbois avec un contrechant de trompette $(1,2: 36-37)$.

FIGURE 9 L'Eau qui danse..., première partie, premier tableau (Tremblay, 2007, p. 27)
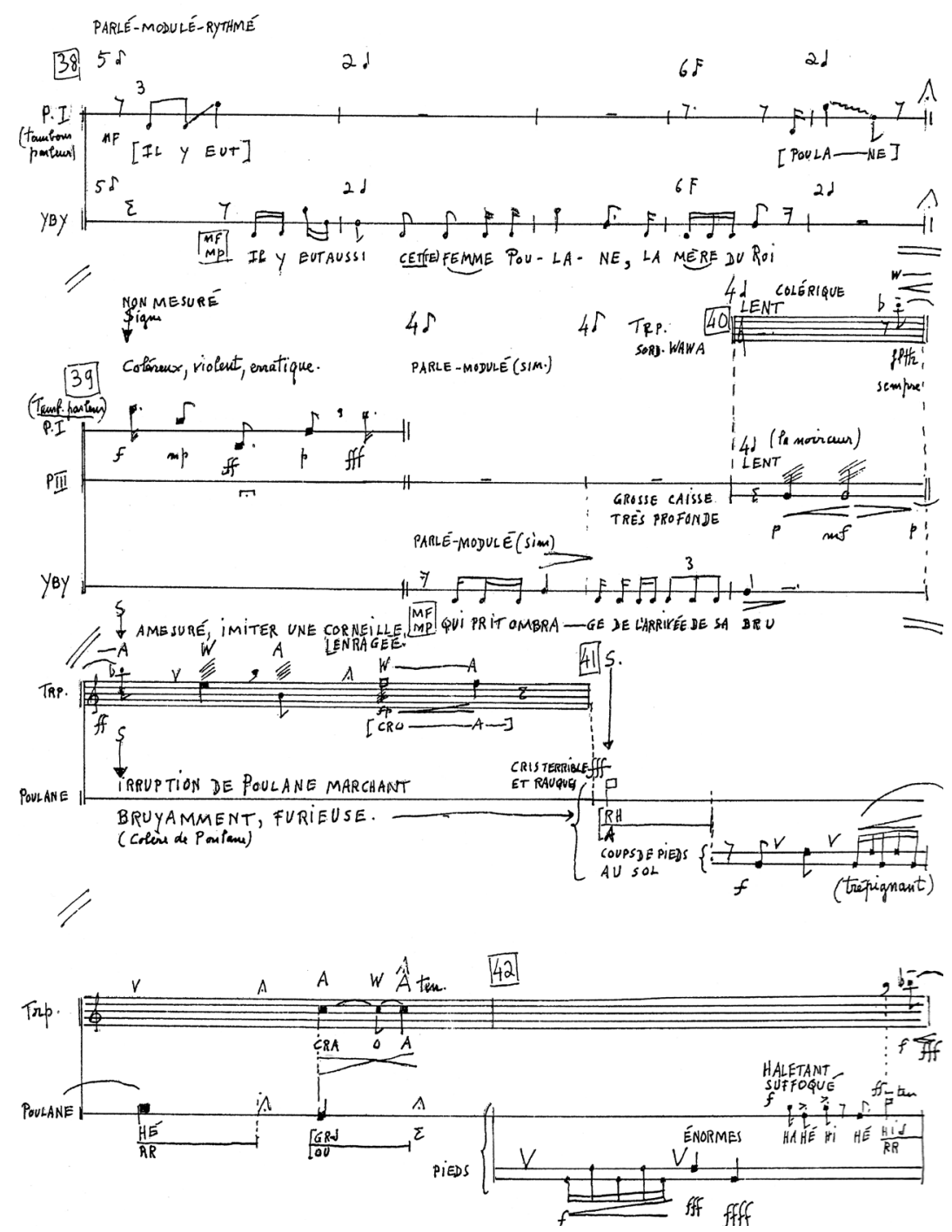

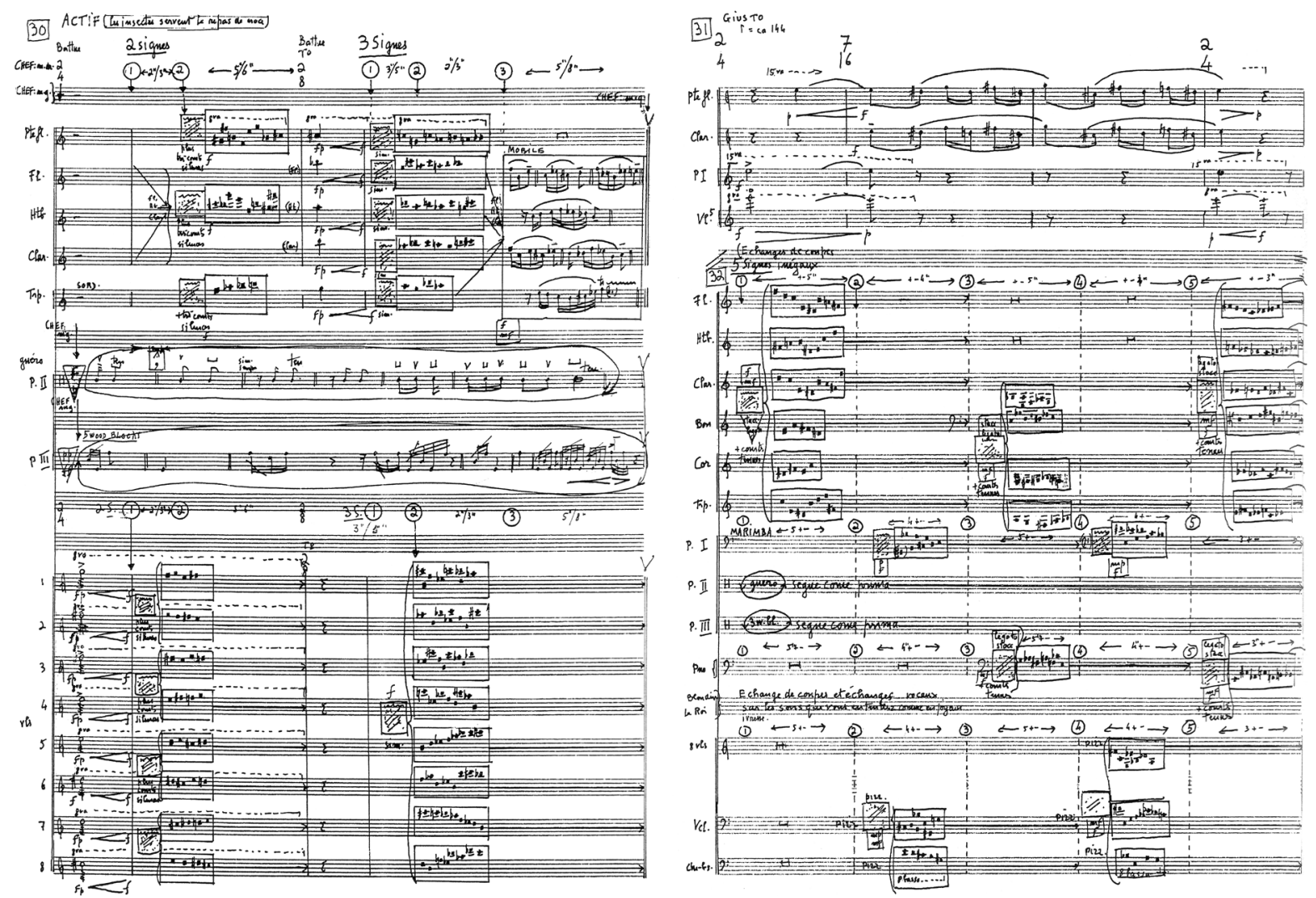

Le conteur Yby et son double, le tambour-parleur, ont pour fonction de lier les événements, les représentations, comme le thème unificateur de la promenade dans les Tableaux d'une exposition de Moussorgski. La présence de ce personnage à la fois extérieur et protagoniste, être hybride, voix hors du temps et de l'action mais qui sait parfois en suivre le cours lorsqu'il se change en passeur $(2,6 ; 2,8)$, rappelle celle du prologue de l'opéra baroque et de sa résurgence à la fin du XIX et au $\mathrm{XX}^{\mathrm{e}}$ siècle. Toutefois, le mode de présence d'Yby est nouveau, jouant le rôle des cartons dans le cinéma muet, offrant 
une cohérence à la dramaturgie du merveilleux, même lorsque le librettiste s'amuse à rajouter un plan de mise en abyme quand Feintise s'enhardit et remarque: «comme on le dit dans les contes» $(2,3: 55)$.

Une étude des symboles vecteurs de merveilleux qui jalonnent l'opéra mériterait à elle seule un ouvrage; prenons à titre d'exemple l'essaim d'abeilles qui entoure les entrées et sorties des protagonistes et dont Yby fait partiellement partie puisqu'il est une créature merveilleuse, mi-abeille, mi-oiseau. Il les interpelle à l'orée de l'opéra : «Premières musiciennes du monde, porteuses d'un chant doré, annonciatrices d'une vérité douce comme le miel et brûlante comme l'aiguille, Vous avez appelé YBY, il arrive, je suis là » (Morency, 2009, p. 3). Or, ces abeilles existent bel et bien dans le conte de Madame d'Aunoy, créatures merveilleuses puisque conjointement musiciennes et servantes, apparues grâce à la fée pour seconder la grandmère dans sa préparation d'un festin pour le Roi :

Le buffet s'arrangea, l'on ne voyait que bassins et que vases d'or, dont le travail surpassait la matière. En même temps un essaim de mouches à miel parut dans des ruches de cristal, et commença la plus charmante musique qui se puisse imaginer. Toute la salle était pleine de frelons, de mouches, de guêpes, de moucherons et d'autres bestiolinettes de cette espèce qui servaient le roi avec une adresse surnaturelle. (d'Aulnoy, 2008, p. 375)

Le compositeur aura rêvé la musique enchantée de ces «bestiolinettes » qui retiennent aussi l'attention du librettiste qui fait s'exclamer Yby: «Venez, arrivez, tous les êtres menus de la nature, abeilles, mouches, guêpes, cigales, grillons, moucherons et bestiolinettes! Chantez la joie neuve d'un roi » (Morency, 2009, p. 3). Le concert des insectes servant les noces du Roi et de Blondine est le premier tutti orchestral de l'œuvre, fonctionnant, comme la plupart de ces tuttis, en juxtaposition de modules mélodiques interprétés librement par chaque groupe d'instruments sous l'autorité du directeur musical (1, 1:30-32).

Mais au-delà de ce concert surnaturel constitutif du conte originel, dans L'Eau qui danse..., les déplacements des personnages dans le temps et dans l'espace sont symbolisés par le bourdonnement d'abeilles suggéré par huit violons au jeu arco et legato, souvent trillé ou en glissando, homorythmique et chromatique. Leur essaim vient nimber puis entraîner le protagoniste ${ }^{29}$.

Si le merveilleux irrigue la partition, tant sur le plan instrumental que vocal, il est cependant indissociable de sa représentation scénique, visuelle, scrupuleusement décrite et notée par le compositeur.
29. Rappelons que Rimsky-Korsakov avait traduit le vol magique du bourdon dans Le Conte du Tsar Saltan.. par un frémissement de cordes devenu morceau d'anthologie. 
FIGURE 11 L'Eau qui danse..., deuxième partie, dixième tableau (Tremblay, 2007, p. 271)

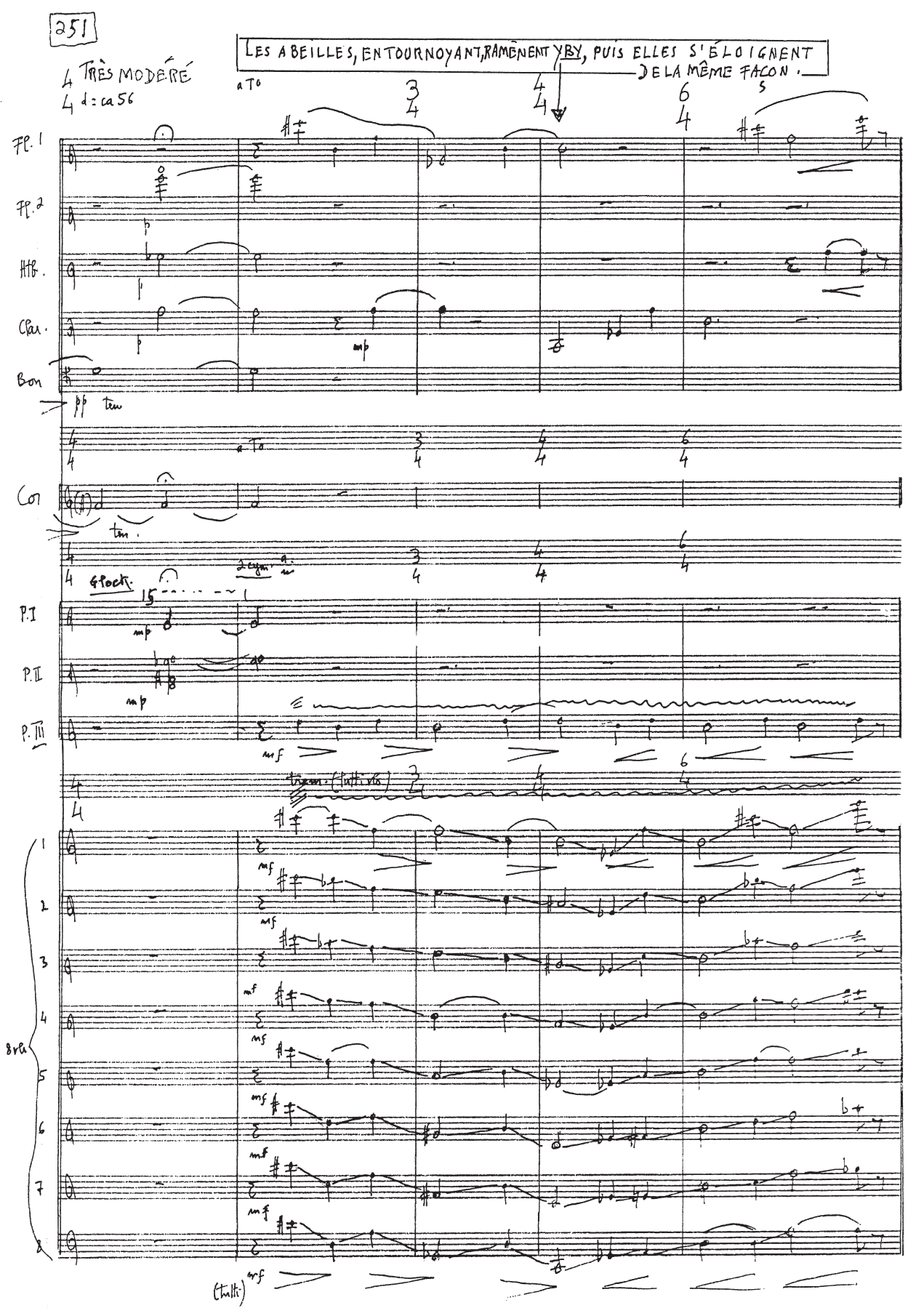




\section{Scénographie du merveilleux en 2009}

Lors de sa création, l'opéra-féerie de Gilles Tremblay et Pierre Morency a été mis en scène par Robert Bellefeuille dont il s'agit de la première expérience d'opéra (Tremblay, Morency, Bellefeuille, Vaillancourt, 2010, «making off»). Menant un «travail archéologique » (ibid.) dans l’œuvre, il laisse éclore texte et musique en une absolue économie de moyens souhaitant conférer à l'image un statut «d'écrin» (ibid.). Nul accessoire, nul décor, des gestes rigoureusement accordés à la psychologie de chaque personnage sont soulignés par les lumières de Nicolas Descoteaux. La scénographie de Jean Bard propose un plateau absolument nu, seulement encadré par les percussions à cour et jardin éclairés a minima, dans un dispositif qui rappelle celui de Noces de Stravinsky. Les costumes de Marianne Thériault et le maquillage d'Angelo Barsetti, en revanche, respectent à la lettre l'esprit symbolique et féerique noté dans la didascalie et la note d'intention qui ouvre la partition: de riches tissus à dominante blanc, or, cuivre et gris, taupe et noir, ainsi que rouge pour la Pomme, des coiffes majestueuses et les plumes de Tourterelle et de l'Oiseau, non seulement présentes sur les costumes, mais aussi au bout de leurs doigts, donnant aux mouvements de leurs mains une grâce singulière rappelant celle des danseuses de Bharatanatyam. Sur un écran occupant tout le fond de scène, les images de Geodezik (Olivier Goulet et Étienne Cantin) rythment les apparitions des protagonistes, offrant un contexte fluctuant de couleurs et d'images abstraites, dialoguant avec les mouvements des artistes.

FIGURE 12 L'Eau qui danse..., troisième partie, deuxième tableau

De gauche à droite: L'Oiseau: Scott Belluz (haute-contre); Beau Jour: Philippe Martel (baryton); le Roi: Taras Kulish (basse); la Reine Blondine: Anne Saint-Denis (soprano); Chérot: Sylvain Paré (ténor) à genoux; la princesse Belle-Étoile: Marianne Lambert (soprano) et dans le coin droit Petit-Soleil: Julien Patenaude (baryton) - Photo: Mathieu Dupuis.

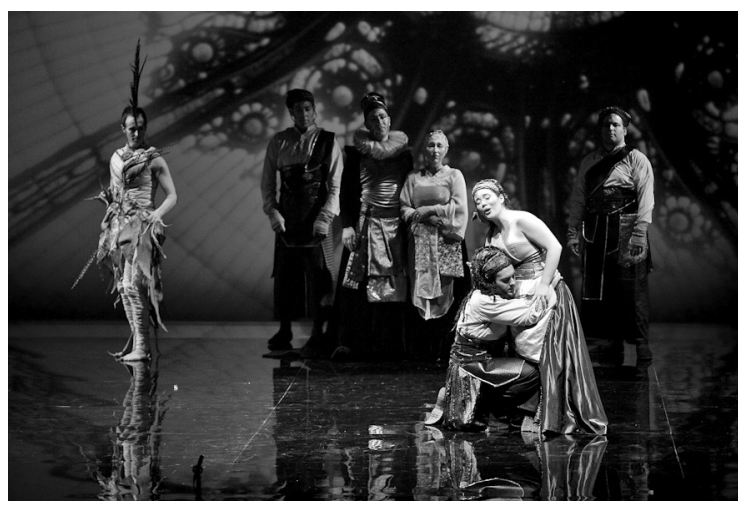


La scène est la plupart du temps plongée dans l'obscurité, ne serait l'écran de fond, bleu dans les scènes positives, vert dans les scènes de trahison, et rouge fluorescent dans la scène de la Pomme. Pour les tableaux de vie et de narration, l'écran est alternativement nu ou rehaussé de la découpe d'une coupole, architecture de fer sur verre inscrite comme une dentelle en ombre chinoise sur la couleur dominante symbolisant le palais.

Les scènes de féerie - la traversée de la forêt, la danse de l'Eau, le combat avec le monstre, la cueillette de la Pomme et l'ascension vers l'Oiseau -, convoquant un merveilleux par essence irreprésentable (Kinztler, 1996, p. 418), sont confiées aux vidéastes de Geodezik pour un traitement multimédia accordé aux subtils jeux de lumières de Nicolas Descoteaux. L'écran projette des images spécifiques, toujours abstraites, mais accordées avec les tons attendus comme le vert-orange-noir et les stries verticales pour la forêt ou les impressions d'éclairs et de crachats de flammes pour le combat avec le dragon suivi de l'éclat quasi insoutenable de l'armure aux mille miroirs tournée vers le public ou encore le bleu glacé des sommets pour la scène de l'Oiseau. La danse de l'Eau, à dominante bleue, est matérialisée par un effet de fumée appliqué aux mouvements d'une danseuse.

La nudité du plateau accentue la portée des gestes et des mouvements des protagonistes accordés à la psychologie de leurs rôles: naturels pour le Roi et sa famille, hachés ou sinueux pour les traîtresses, hiératiques pour Yby, d'un dépouillement chorégraphique pour les personnages merveilleux.

FIGURE 13 L'Eau qui danse..., troisième partie, deuxième tableau

De gauche à droite: Beau Jour: Philippe Martel; Feintise: Michèle Motard; Poulane: Marie-Annick Béliveau; Chérot: Sylvain Paré; le Roi : Tarash Kulish; Belle-Étoile: Marianne Lambert; Blondine: Anne St-Denis; Petit Soleil: Julien Patenaude; la Pomme: Claudine Ledoux $•$ Photo: Mathieu Dupuis.

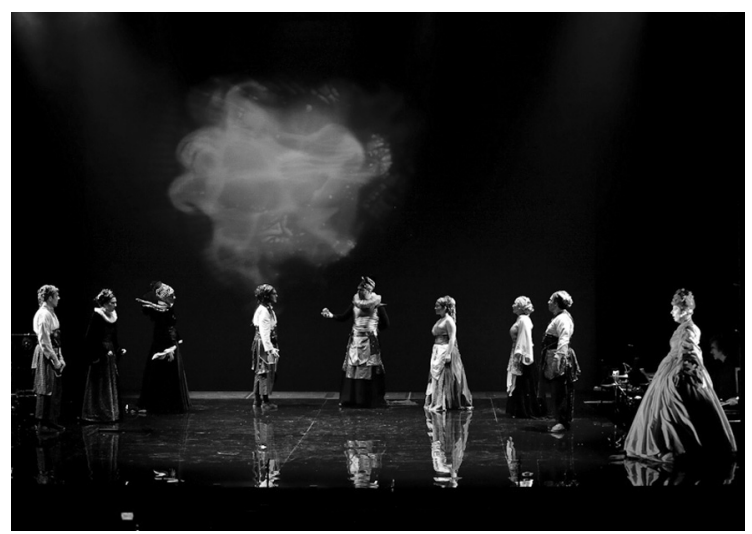


Cette économie de moyens héritée de Bob Wilson favorise le déploiement de toute la richesse de la partition musicale de L'Eau qui danse... dans l'esprit des mises en scène féeriques de Wilson: La Flûte enchantée, La Femme sans ombre ou encore les Fables de La Fontaine. Dans cet esprit de dépouillement, Robert Bellefeuille a cependant privé Gilles Tremblay de la visualisation de scènes qu'il avait imaginées, notées, voire chorégraphiées.

En effet, le compositeur demande qu'un acrobate soit engagé pour mimer les épreuves de Chérot, idée abandonnée dans la mise en scène de la création, comme celle des gestes statiques du théâtre Nô pour les scènes d'affrontement entre Poulane et Feintise, plus développées par Robert Bellefeuille tandis que les banderoles qui devraient accompagner la danse de Tourterelle $(2,2)$ sont elles aussi abandonnées. Le chaste et fraternel baiser de la première «bulle des amoureux» se transforme en scène d'une grande sensualité.

Enfin, les indications qui placent les tableaux de l'Oiseau (2, 9-10) dans une vision quasi baroque du merveilleux sont transcendées par Robert Bellefeuille.

L'Oiseau apparaît, découpe blanche emplumée sur l'écran de fond bleu et noir, les deux couleurs étant réparties de part et d'autre d'une diagonale légèrement incurvée allant du coin bas jardin au coin haut cour. Le triangle noir partant du sol symbolise le rocher qui renferme les frères de Belle-Étoile et les autres chevaliers. Dans cette scène, la joute sera uniquement verbale, car il ne s'agit plus pour Chérot de se mesurer à son courage ou à sa force, mais plutôt de réfléchir et de déployer ruse, intelligence et bienveillance. L'image de l'Oiseau est d'une très grande beauté, renforcée par les sonorités harmoniquement dissonantes mais timbriquement homogènes des instruments, par exemple l'alliance glockenspiel-vibraphone-cloches-piano (2, 9: 190-192). Le seul effet de lumière consiste ici à jouer sur l'éclairage des corps pour les faire apparaître en totalité ou en ombres chinoises. Le spectateur peut alors pleinement goûter la subtilité des timbres et la beauté du texte.

L'utilisation d'une palette instrumentale très variée, en particulier des percussions, de multiples émissions vocales et le recours à une scénographie innovante bien que minimaliste s'inscrit naturellement dans la logique de l'ouverture de la musique dite savante à toutes les influences, sans que l'on puisse ici parler de métissage. Comme d'autres compositeurs d'opéra, Graciane Finzi $3^{\circ}$ en particulier, Tremblay puise à de multiples sources pour construire son univers artistique adossé aussi bien à la musique qu’à la poésie ou à la contemplation et l'empathie avec la nature et la vie et dont son opéraféerie marque sans doute un point d'aboutissement en dépassant l'abstraction instrumentale grâce à l'alliance de la poésie et des arts de la scène.
30. www.graciane-finzi.com/ 


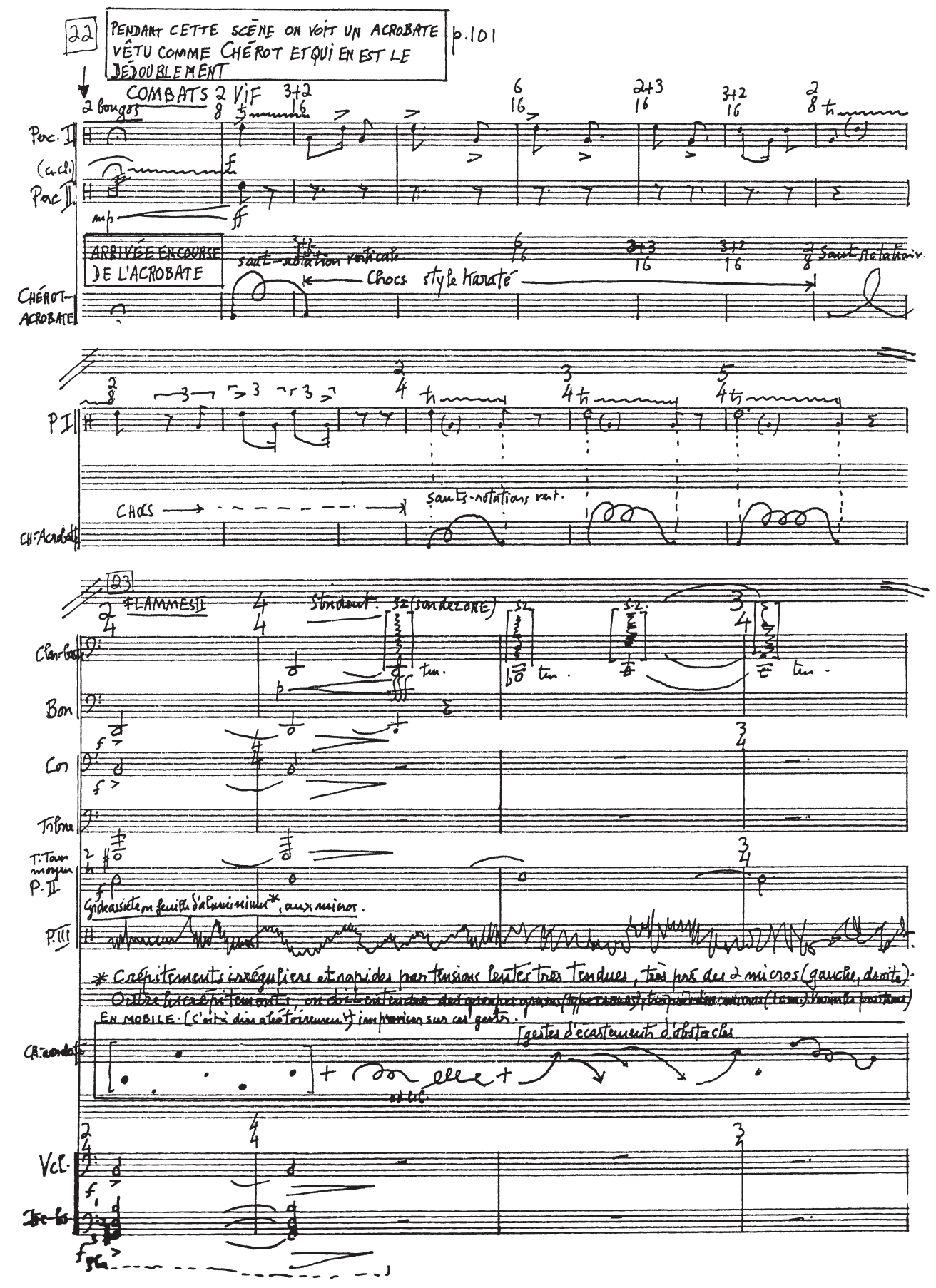


p.223

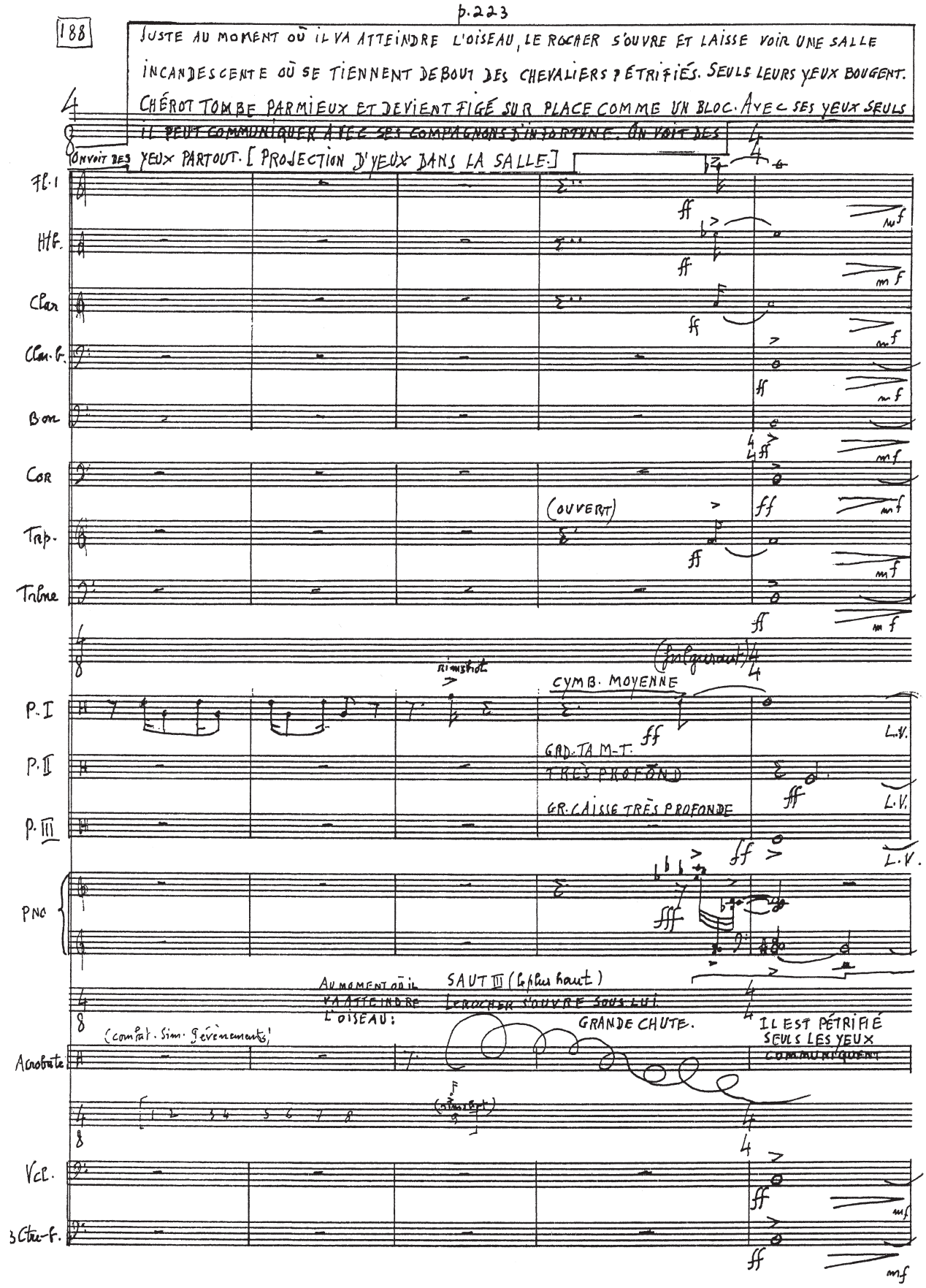


31. Nous traduisons: "All in all, the conteuses use the fairy-tale form to create a counterideology in which women assert their own abilities and desires to participate in cultural and, especially, literary production".

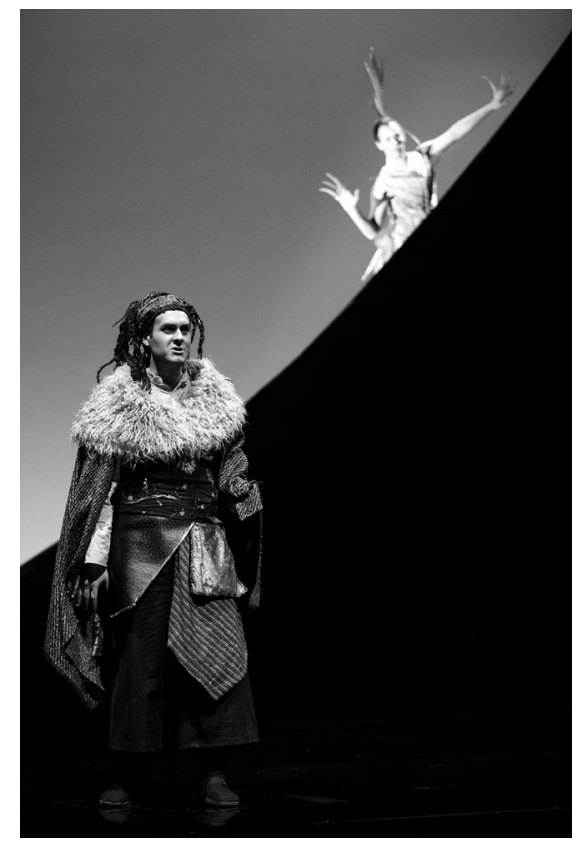

\section{Par-delà la nostalgie, "le merveilleux existe"}

Venu de la tradition orale, puis fixé mais sans cesse réécrit (Velay-Vallantin, 1992, p. 11-40), comme nous le prouve l'histoire de la princesse Belle-Étoile, le conte s'adapte aux contextes culturels les plus variés par sa portée archétypique. L'Eau qui danse... offre-t-elle une adaptation postmoderne du conte de Madame d'Aulnoy?

Si la mode des conteuses à la fin du XVII siècle avait pour but de «créer une contre-idéologie dans laquelle les femmes prouvent leurs compétences et désirent participer aux productions culturelles, en particulier dans le domaine de la littérature » (Seifert, 2001, p. 932 $2^{31}$ ), à ce titre, Madame d'Aulnoy aurait offert dans ses contes une alternative à la domination masculine prônée par les opéras de Quinault et Lully (Duggan, 2004, p. 302). En poursuivant l'interrogation de Michel Leiris: «Coupant court à tout discours, le merveilleux peut-il être discoureur?» (1992, p. 75), on peut alors se demander si Gilles Tremblay et Pierre Morency cherchent à s'insérer aussi dans une contre-culture en proposant une forme de réenchantement de la scène lyrique. 
En effet, prendre de la distance avec l'opéra politique ou social en renouant avec une forme de distanciation du réel ne permet-il pas, par la catharsis, de mieux reprendre pied dans l'existence? Car en se demandant si «un progrès épistémologique est [...] un progrès poétique », Catherine Kintzler (1997, p. 409) pose une autre juste question: que devient le droit de rêver quand une société cherche à tout prix à objectiver le monde, à le circonscrire, que ce soit par la raison à la fin du XVIII ${ }^{\mathrm{e}}$ siècle ou par l'information à la fin du $\mathrm{XX}^{\mathrm{e}}$ ? N'est-ce pas pour échapper à l'inflation du matérialisme que le thêâtre lyrique s'échappe aujourd'hui à nouveau vers les cimes oniriques d'un imaginaire qui revendique sa libre expression?

Dans le monde souvent désenchanté des opéras de ces dernières décennies, stigmatisant la vanité de la civilisation occidentale dont l'opéra est tout de même le produit, les compositeurs stimulés par la scène lyrique se tournent parfois vers le conte ${ }^{32}$ et la démarche des artistes de L'Eau qui danse... s'inscrit dans cet élan de la subjectivité qui n'avait pas échappé à Gilbert Durand au lendemain de Mai 68. Selon lui, la multiplication des expériences mystiques ou hallucinées s'oppose à l'art abstrait: «Notre civilisation rationalise et son culte pour la démystification objective se voit submergé en fait par le ressac de la subjectivité brimée et de l'irrationnel » (Durand, 1969, p. 495), générant, parmi d'autres, la réaction ornithologique de Messiaen à l'intellectualisation artistique, tout comme l'attitude d'écoute de Gilles Tremblay à l'égard de la nature, de l'univers et des liens merveilleux qui s'y tissent.

Ainsi, la scène lyrique de Tremblay et Morency, puisant ses racines dans les mythes, donc dans l'imaginaire et, partant, dans l'inconscient, invite à poser un regard bienveillant sur l'être humain et ses désirs. En s'appuyant sur les conventions du genre tout en les renouvelant, les artistes ont imaginé un spectacle à la fois divertissant, onirique et cathartique, renouant avec les principes spectaculaires fondateurs des cultures humaines en les adaptant aux langages poétique, musical et visuel de leur temps. Prototype de l'écrivaine aristocratique dont la conversation surpasse encore les textes, Madame d'Aulnoy, comme Gilles Tremblay, produit une œuvre qui n'est finalement qu'une partie infime de son être, renvoyant le lecteur-spectateur au réel une fois l'œuvre terminée: où peut-on trouver, dans le monde, un écho au merveilleux?

À ce titre, la démarche de Tremblay, Morency et Bellefeuille s'accorde avec la contre-idéologie définie par Seifert (2001, p. 932) car L'Eau qui danse... apparaît comme une alternative au Regietheater, à l'opéra à grand spectacle, ou aux sujets d'histoire, d'actualité. Sa conclusion offre un écho à la constatation de Marie-Jeanne Lhéritier en 1696 au sujet de la mode
32. Dans les vingt dernières années, citons: Unsuk Chin: Alice in Wonderland (Munich, 2007), Patrick Burgan: Peter Pan (Paris, 2006), Pierangelo Valtinoni : Pinocchio (Berlin, 2006), Hans Werner Henze: L'Upupa und der Triumph der Sohnesliebe (Salzbourg, 2003), Rachel Portman: The Little Prince (Houston, 2003), Alexander Knaifel: Alice in Wonderland (Amsterdam, 2001), Heinz Holliger: Schneewittchen (Zurich, 1998), Sergio Menozzi : Pinocchio (Lyon, 1998), Wilfried Hiller: Peter Pan (Munich, 1997), Marco Tutino: // gatto con gli stivali (Vérone, 1997), Giampaolo Testoni : Alice (Palerme, 1993). 
33. En cinq strophes matérialisées par des chiffres romains dans le texte ci-dessus cité

34. Qui avouait au sujet de ses lectures d'enfant: "J'aurais pu tirer un livret d'opéra de ces contes, par exemple du conte persan où le cordonnier Azbiaze devient roi" (Massin, 1989, p. 26). des contes de fées: "On voit de petites Histoires répandues dans le monde dont tout le dessein est de prouver agréablement la solidité des proverbes » (ibid., p. 912). La Princesse Belle-Étoile s'achève en effet par une morale «[...] Mais un cœur ne craint pas les plus grands précipices / S’il a pour l'animer, et la gloire, et l'amour » (Aulnoy, 2001, p. 431). Trois siècles plus tard, Pierre Morency (2009, p. 31) épouse cette démarche tout en l'ouvrant vers l'imaginaire, tendant le miroir au spectateur à travers la dernière vérité de l'Oiseau, qui convainc le Roi de lui rendre la liberté:

[I] La vérité de cette histoire est qu'à chaque vie humaine Apparait toujours une occasion d'induire la lumière.

[II] Le merveilleux existe et n'a pas d'autres couleurs

Que celle que l'on porte dans le cristal de soi-même.

[III] Le plus clair de l'existence est cette liberté

Que donnent le lien d'amour et la découverte des choses les plus simples.

[IV] Vérité grande est cette liberté que l'on donne à l'Oiseau

De chanter la pure beauté d'être vivant.

[V] Ce qui s'élève pour le monde

L'Oiseau le voit car il le chante

Ce qui s'élève dans le chant

C'est pour le monde une soif d'enchantement.

(Livret de Morency pour Tremblay, 2010, p. 146)

Ce «dernier poème de l'Oiseau33» (Tremblay, 2010, p. 146) est chanté $a$ capella; entre chaque strophe revient la musique de voyelles de la Pomme et la danse de l'Eau, ensemble ou séparément. L'Oiseau qui dit la vérité apparaît alors comme un reflet double des oiseaux musiciens de Messiaen, mais cette fois, prenant comme son maître appui sur le Merveilleux (Massin, 1989, p. 27-28), Tremblay transcende l'abstraction du modèle sonore naturel et donne la parole à l'Oiseau, à l'Oiseau-Prophète en écho également à Schumann pour donner aux hommes les clés d'un univers dont il convient de lire le langage implicite, comme nous y invite Bachelard (1942, p. 22):

$[\ldots]$ le langage des eaux est une réalité poétique directe, $[\ldots]$ les ruisseaux et les fleuves sonorisent avec une étrange fidélité des paysages muets, [...] les eaux bruissantes apprennent aux oiseaux et aux hommes à chanter, à parler, à redire, et [...] il y a en somme continuité entre la parole de l'eau et la parole humaine.

N'est-il pas plus bel hommage de Gilles Tremblay à l'enseignement musical, mystique et humain de son maître Olivier Messiaen34 que d'ouvrir ainsi l'une des voies du renouveau de l'art lyrique grâce à la puissance de l'imaginaire nourri de la contemplation et de l'émerveillement devant le monde réel? Car «Pour le musicien, entendre, percevoir les relations musicales qui sont dans la vie, constitue déjà un acte musical. [...] Si l'œuvre aide 
l'auditeur à son tour à percevoir les musiques latentes qui nous entourent, alors le musicien sera comblé» (Tremblay, 1994, p. 9).

\section{BIBLIOGRAPHIE}

Aulnoy, Madame d’ (2008), «La Princesse Belle-Étoile et le Prince Chéri », Contes nouveaux ou Les Fées à la mode (1698), Nadine Jasmin (dir.), Paris, Honoré Champion, p. 371-431.

Auzolle, Cécile (1995), Études pour une esthétique de la fête dans le théatre lyrique depuis Les Noces de Figaro de Mozart, thèse de nouveau doctorat, Université Paris-Sorbonne Paris-IV, Lille, Atelier National de Reproduction des Thèses, 1995.

BaChelard, Gaston (1942), L'Eau et les rêves, essai sur l'imagination de la matière, Paris, Corti.

Bettelheim, Bruno (1976), Psychanalyse des contes de fées, Paris, Laffont.

Biget-Mainfroy, Michelle (2001), «Quand les compositeurs relisent les contes », Il Saggiatore musicale, vol. viII, $\mathrm{n}^{\circ}$ 2, p. 241-259.

Borvin, Jean (1995), La Classe de Messiaen, Paris, Bourgois.

Borvin, Jean (2003), «Musique et nature», Musiques, une encyclopédie pour le XXI siècle, Vol. 1: Musiques du XXe siècle, Jean-Jacques Nattiez (dir.), Arles, Actes Sud, p. 484-511.

Bouchard, Alfred (1878), La Langue théatrale: vocabulaire historique, descriptif et anecdotique des termes et des choses du théâtre, suivi d'un appendice contenant la législation théatrale en vigueur, Paris, Arnaud et Labat.

Buch, David J. (1992), «Fairy-tale Literature and Die Zauberflöte», Acta musicologica, vol. 64, $\mathrm{n}^{\circ}$ 1, p. 30-49.

Carrington, John F. (1971), «The Talking Drums of Africa », Scientific American, vol. 225, nº 6, p. 90-94.

Christout, Marie-Françoise (1982), «La Féerie romantique au théâtre: de La Sylphide (1832) à La Biche au bois (1945). Chorégraphies, décors, trucs et machines ", Romantisme: revue de la Société des Études Romantiques, nº 38, p. 77-86.

Duggan, Anne E. (2004), «Women and Absolutism in French Opera and Fairy Tale», The French Review vol. 78 n ${ }^{\circ}$ 2, p. 302-315.

Durand, Gilbert (1992), Les Structures anthropologiques de l'imaginaire (1969), Paris, Dunod.

JASmin, Nadine (2008), «Introduction", Madame d'Aulnoy, Contes nouveaux ou les fées à la mode, Paris, Honoré Champion, p. 7-24.

KinzTLER, Catherine (1996), «Du merveilleux et du sublime: nature lacunaire, surnature, contre-nature ", D’un opéra l'autre, Hommage à Jean Mongrédien, Jean Gribenski (dir.), Paris, Presses Universitaires de la Sorbonne, p. 413-420.

KinzTLER, Catherine (1997), «La poétique du merveilleux dans l'opéra français et son effondrement à la fin du XVIII e siècle», Mozart: les chemins de l'Europe, Brigitte Massin (dir.), Strasbourg, Éditions du Conseil de l'Europe, p. 405-413.

KinZTLER, Catherine (2002), «L'opéra, théâtre ou spectacle? », Récit et représentation musicale, Cohen-Levinas, Danielle (dir.), Paris, L'Harmattan, p. 115-124.

LeIrIs, Michel (1992), Operratiques, Paris, P.O.L.

Massin, Brigitte (1989), Olivier Messiaen, une poétique du merveilleux, Aix-en-Provence, Alinéa.

Messiaen, Olivier (1971), «Préface» J. Chaix-Ruy, Du féerique au céleste, Grenoble, Roissard, p. 7-9.

Miquel, André (2005), «Préface», Les Mille et une nuits, Paris, Gallimard, coll. «Bibliothèque de la Pléiade», vol. 1, p. XI-XLVI.

Morency, Pierre [2009], Opéra féerie, L'Eau qui danse, la pomme qui chante et l'oiseau qui dit la vérité, livret, Montreal, Chants libres.

Owomoyela, Oyekan (1993), «Drums in african folklore», Drums: The Heartbeat of Africa, Esther A. Dagan (dir.), Montreal, Galerie Amrad African Art Publications, p. 58-61. 
Seifert, Lewis (2001), «The Marvelous in context: The Place of the Contes de Fées in Late Seventeenth-Century France (1996)", in The Great Fairy Tale Tradition From Straparola and Basile to the Brothers Grimm, Jack Zipes (dir.), New York/Londres, Norton, p. 902-933.

Tremblay, Gilles [2010], Opéra féerie, L'Eau qui danse, la pomme qui chante et l'oiseau qui dit la vérité, réduction pour voix et piano [Montréal], Centre de Musique Canadienne.

Tremblay, Gilles (2007), Opéra féerie, L'Eau qui danse, la pomme qui chante et l'oiseau qui dit la vérité, partition d'orchestre manuscrite [photocopie confiée par la Compagnie Chants libres].

Tremblay, Gilles (1995), «Transcription d'une écorce de bouleau », Circuit vol 6 nº 1, p. 61-63.

Tremblay, Gilles (1994a), «L'acte musical (1970)», Circuit vol. 5 nº 1, p. 9.

Tremblay, Gilles (1994b), "Composer de la musique contemporaine (1973)», Circuit vol. 5 $\mathrm{n}^{\circ}$ 1, p. 10-17.

Tremblay, Gilles (1970), «Dimension soleils », Revue de la Société de musique contemporaine du Québec.

Tremblay, Gilles, Morency, Pierre, Bellefeuille, Robert (2009), Opéra féerie, L'Eau qui danse, la pomme qui chante et l'oiseau qui dit la vérité, programme, Montréal, Chants libres.

Tremblay, Gilles et Duchesnay, Gilles (1983), Fascicule imprimé de l'Anthologie de la musique canadienne, Gilles Tremblay, ACM 12. 6 LP, Radio Canada International.

Velay-Vallantin, Catherine (1992), L'Histoire des contes, Paris, Fayard.

Waters, W. G. (2001), « [The Mysterious Giovan Francesco Straparola, Founding Father of the Fairy Tale] (1891)», in Zipes (dir.), 2001, op. cit., p. 869-879.

Yon, Jean-Claude (2006), «La Féerie ou le royaume du spectaculaire: l'exemple de Rothomago », Isabelle Moindrot (dir.), Le Spectaculaire dans les arts de la scène: du romantisme à la Belle époque, Paris, CNRS.

Zipes, Jack (2001), The Great Fairy Tale Tradition From Straparola and Basile to the Brothers Grimm, New York/Londres, Norton.

\section{Sites INTERNET DE RÉfÉRENCE (CONSUltéS AU PRINTEMPS 2010) :}

\section{Gilles Tremblay:}

http://www.smcq.qc.ca/smcq/fr/artistes/t/tremblay_gi/

http://www.smcq.qc.ca/smcq/fr/artistes/t/tremblay_gi/oeuvres.php

http://www.radio-canada.ca/espace_musique/webRadioClassique.asp\#section=magazine\&idRu brique $=128680$

\section{Graciane Finzi :}

http://www.graciane-finzi.com/

Sources des contes de Pouchkine:

http://feb-web.ru/febit/pushkin/serial/vr1/vr12134-.htm

Tambour parleur:

http://vivrelemonde.free.fr/instrumentarium.php

\section{DISCOGRAPHIE, VIDÉOGRAPHIE :}

Tremblay, Gilles (1983), ACM 12. 6 LP, Anthologie de la musique canadienne, Radio Canada International.

Tremblay, Gilles, Morency, Pierre, Bellefeuille, Robert, Vaillancourt, Pauline (2010), L'Eau qui danse, la Pomme qui chante et l'Oiseau qui dit la vérité, opéra-féerie, [captation du spectacle sur DVD], Montréal, Compagnie lyrique de création Chants Libres/Gaspacho films. 